\title{
Whipping IC 63/IC 59
}

\author{
H. Andrews ${ }^{1}$, E. Peeters ${ }^{2,3}$, A. G. G. M. Tielens ${ }^{1}$, and Y. Okada ${ }^{4}$ \\ ${ }^{1}$ Leiden Observatory, Leiden University, PO Box 9513, 2300 RA Leiden, The Netherlands \\ e-mail: tielens@strw.leidenuniv.nl \\ 2 Department of Physics and Astronomy, University of Western Ontario, London, ON N6A 3K7, Canada \\ e-mail: epeeters@uwo.ca \\ ${ }^{3}$ Carl Sagan Center, SETI Institute, 189 N. Bernardo Avenue, Suite 100, Mountain View, CA 94043, USA \\ ${ }^{4}$ Physikalisches Institut der Universität zu Köln, Zülpicher Straße 77, 50937 Köln, Germany \\ e-mail: okada@ph1.uni-koeln.de
}

Received 11 February 2018 / Accepted 4 September 2018

\begin{abstract}
Context. IC 63 and IC 59 are a pair of cometary-shaped nebulae in the vicinity of the star $\gamma$ Cas (also known as Tsih, "the Whip"). Both nebulae have very different optical appearances, despite the fact that both objects lie at similar projected distances from the star: IC 63 shows bright rims and filaments, while IC 59 looks more homogeneous and faint.

Aims. We aim to perform a general description of the two nebulae from an observational standpoint in order to study the physical conditions at the UV-illuminated surfaces of these objects.

Methods. We used the available data on both nebulae taken with Spitzer and Herschel to study the infrared emission at the tip of both clouds, and derive the intensity of the UV radiation field. Using the photodetector array camera and spectrometer (PACS) in line spectroscopy mode, we obtained the intensity of the cooling lines [C II] $157 \mu \mathrm{m}$ and [O I] $63 \mu \mathrm{m}$, and we used these to estimate the density in these regions. Excitation diagrams of molecular hydrogen were obtained to derive the gas temperature. We also used [C II] $157 \mu \mathrm{m}$ velocity maps of IC 59 taken with German REceiver for Astronomy at Terahertz frequencies (GREAT) on board SOFIA to explore the gas dynamics in this nebula.

Results. We find that the IR emission from polycyclic aromatic hydrocarbons (PAHs) is very similar at the tip of both nebulae. Even though it varies in intensity between the two, the derived PAH band ratios are remarkably similar. These ratios are similar to those found in the more shielded regions of other nebulae such as NGC 7023 and NGC 2023. Regarding the physical conditions, we get that while in IC 63 the intensity of the UV field, $G_{0}$, is a factor of approximately ten higher than in IC 59, the density $n$ at the tip of IC 59 is lower than in IC 63 by a similar factor. For both objects we derive $G_{0}$ values significantly lower than what previous works have so far assumed. Comparison with other reflection nebulae photo-dissociation regions (PDRs) and known correlations support our claim that both IC 63 and IC 59 are low-UV irradiated environments.

Conclusions. We conclude that the tips of IC 63 and IC 59 are about three and five times farther away from the star than their respective projected distances. The similarity of the mid-infrared emission between the two nebulae is consistent not only with both objects being overdensities within the same region around $\gamma$ Cas, but it is also consistent with the similar $G_{0} / n$ and ionization parameters, which altogether rule the evolution of the hydrogenation and ionization level of the emitting population of PAHs. Finally, regarding the kinematics of the material in IC 59, we find evidence of photo-evaporation due to the incident radiation from $\gamma$ Cas.
\end{abstract}

Key words. astrochemistry - photon-dominated region - ISM: molecules - infrared: ISM

\section{Introduction}

Polycyclic aromatic hydrocarbons (PAHs) are presumed to be the most abundant organic molecules in space, with abundances on the order of $10^{-7}$ to that of hydrogen. They are a set of very stable planar molecules disposed in a carbon core with hydrogen atoms attached to their periphery (Léger \& Puget 1984; Allamandola et al. 1985). After the absorption of high energy $(<13.6 \mathrm{eV})$ photons, these molecules relax through their vibrational modes emitting copiously in the IR, and producing strong emission bands at $3.3,6.2,7.7,11.3$, and $12.6 \mu \mathrm{m}$ together with weaker bands. Therefore, their emission is the brightest in regions illuminated by UV radiation, such as photon-dominated regions (PDRs).

Photon-dominated regions can generally be described as the regions of the interstellar medium (ISM) where the photochemistry is dominated by UV radiation (Hollenbach \& Tielens 1997; Hollenbach \& Tielens 1999). In the clouds around young hot stars, the PDRs correspond to the transition zones between the ionized (mostly atomic) material, and the cold molecular clouds. They are regions of (mostly neutral) gas and dust, where we see the transition from atomic $\mathrm{H}$ to molecular $\mathrm{H}_{2}$ (and from $\mathrm{C}^{+}$to $\mathrm{C}$ to $\mathrm{CO}$ ). These interface regions are thus ubiquitous in space. Hence, understanding the mechanisms that rule their evolution is of great importance, as these regions are where the exchange of energy between the stars and the ISM occurs.

The physical conditions within a PDR mainly depend on two parameters: the intensity of the UV field $G_{0}$, and the total density $n$. The main heating mechanisms within a PDR are the photoelectric effect on PAHs and small dust grains, and the FUV pumping of $\mathrm{H}_{2}$ molecules. Being the smallest particles in the dust grain distribution, PAHs are particularly efficient in heating the gas through the photoelectric effect (D'Hendecourt \& Léger 1987; Lepp \& Dalgarno 1988; Bakes \& Tielens 1994). Deeper in the cloud other heating mechanisms become more relevant, such as photoionization of elements, cosmic ray ionization and gas-grain collisions, among others (Tielens \& Hollenbach 1985; Hollenbach \& Tielens 1999). On the other hand, the main 
cooling mechanism in PDRs is due to radiative line cooling of forbidden transitions, such as [C II] $157 \mu \mathrm{m}$ and [O I] $63 \mu \mathrm{m}$.

Photon-dominated regions (PDRs) are then bright in IR emission due to the emission from vibrational modes of PAHs, rotational and vibrational lines of $\mathrm{H}_{2}$, fine structure lines (e.g., [C II], [O I] $)$, rotational lines of $\mathrm{CO}$, and dust continuum emission. Observations of the near, mid and far-IR emission of PDRs obtained with the Spitzer Space Telescope (Werner et al. 2004) and Herschel Space Observatory (Pilbratt et al. 2010) allow us to derive the physical conditions in these regions. In this work, we use photometric and spectroscopic data from Spitzer and Herschel to study the physical conditions in the pair of nebulae IC 63 and IC 59, both in the vicinity of the star $\gamma$ Cas. We also include velocity maps from the Stratospheric Observatory For Infrared Astronomy (SOFIA; Young et al. 2012) to study the dynamics of the material in IC 59.

IC 63 and IC 59 are a pair of nebulae in the constellation of Cassiopeia (IC $59 \mathrm{~J} 2000.0$ coordinates: $00^{\mathrm{h}} 57^{\mathrm{m}} 34^{\mathrm{s}},+61^{\mathrm{d}} 06^{\mathrm{m}} 15^{\mathrm{s}}$; IC 63 J2000.0 coordinates: $\left.00^{\mathrm{h}} 59^{\mathrm{m}} 00^{\mathrm{s}},+60^{\mathrm{d}} 53^{\mathrm{m}} 14^{\mathrm{s}}\right)$. Both objects are illuminated by the B0 IV star $\gamma$ Cas (also referred to as HD 5394 or $T s i h$, the Whip). The projected distance between IC 63 and $\gamma$ Cas is about 20 arcmin or $\sim 1.3 \mathrm{pc}$, while the projected distance between IC 59 and the star is about $1.5 \mathrm{pc}$. Observations in $\mathrm{H} \alpha$ show the system is embedded in a larger diffuse cloud of about 2 degrees surrounding $\gamma$ Cas (Karr et al. 2005).

The PDR of IC 63 has been extensively studied both theoretically and observationally. In particular, the works of Jansen et al. (1994, 1995, 1996) have positioned IC 63 as a warm and high-density PDR, with molecular abundances similar to those found in dark clouds, and with a very low gas-phase carbon abundance (only 13\% that of the solar abundance). Excitation diagrams of $\mathrm{H}_{2}$ obtained with ISO data showed two components in IC 63 of 106 and $685 \mathrm{~K}$ and column densities of $5.9 \times 10^{21}$ and $1.2 \times 10^{19} \mathrm{~cm}^{-2}$, respectively (Thi et al. 2009). Physical conditions including $\mathrm{H}_{2}$ column density and $G_{0} / n_{\mathrm{H}}$ as well as the gas temperature, have been found to vary only slightly at the tip of IC 63 (Karr et al. 2005; Thi et al. 2009; Fleming et al. 2010).

IC 59 on the other hand has been less studied. Physical conditions derived from ISO data reveal a slightly cooler and less dense object than IC 63, but also show $\mathrm{H}_{2}$ fluorescence and $\mathrm{PAH}$ emission just like in IC 63 (Karr et al. 2005). This suggests that, although both nebulae appear to be at a similar projected distance to the star, they are actually at very different real distances, where IC 59 is probably the farthest object away from $\gamma$ Cas.

Regarding PAH emission, 5.5-17.0 $\mu \mathrm{m}$ ISOCAM CVF spectra of both nebulae suggest similar PAH populations at the tip of both objects (Karr et al. 2005). Fleming et al. (2010) focused on the PAH emission at the tip of IC 63 only, and using the low resolution Spitzer spectra, they found that the $\mathrm{PAH}$ and $\mathrm{H}_{2}$ emissions appear to come from the same parts of the nebula. They also found that the dehydrogenation ratio (as measured from the intensity ratio between the PAH features at 12.7 and $11.3 \mu \mathrm{m})$ remains rather constant throughout most of the studied region, decreasing only slightly near the starward edge of the PDR. No PAH emission study from Spitzer spectral data has been published so far on IC 59.

The goal of this paper is to analyze the PAH emission and the physical conditions in both IC 63 and IC 59 from an observational perspective, using data from the Spitzer, Herschel and SOFIA observatories. Section 2 presents the Spitzer, Herschel, and SOFIA data. Section 3 shows first the analysis of the PAH emission, followed by the determination of the physical conditions in both nebulae. In Sect. 4, we analyze what the physical conditions imply about the PAH emission, and their role in the heating of the gas in these two nebulae. We also discuss the dynamics in IC 59 based on the SOFIA [C II] $157 \mu \mathrm{m}$ velocity maps obtained for this cloud. Finally, Sect. 5 presents the conclusions of this work.

\section{Data}

\subsection{Spitzer data}

\subsubsection{IRAC and MIPS photometry}

We used photometric data on both nebulae taken with the InfraRed Array Camera (IRAC; Fazio et al. 2004) and the multiband imaging photometer for Spitzer (MIPS; Rieke et al. 2004). The data were retrieved from the Spitzer heritage archive as part of the program ID 202 for IC 63 (PI: G. Fazio) and ID 3512 for IC 59 (PI: C. Joblin). The raw data products of the IRAC images at 3.6, 4.5, 5.8, and $8.0 \mu \mathrm{m}$, and MIPS images at $24 \mu \mathrm{m}$ were processed with the Spitzer science centre (SSC) pipeline. Corrections for extended emission, zodiacal light and background subtraction were performed on all images. The IRAC and MIPS photometry are accurate to $10 \%$. The full-width-athalf-maximum (FWHM) of the point-spread function (PSF) of the IRAC images is of $1.6^{\prime \prime}, 1.6^{\prime \prime}, 1.8^{\prime \prime}$, and $1.9^{\prime \prime}$ at 3.6, 4.5, 5.8, and $8.0 \mu \mathrm{m}$, while that of MIPS $24 \mu \mathrm{m}$ is of $6^{\prime \prime}$.

\subsubsection{IRS spectra}

We retrieved the spectroscopic data for both IC 63 and IC 59 from the Spitzer heritage archive. The basic calibrated data (BCD) set is part of the SPECPDR program (ID: 3512, PI: C. Joblin), and was obtained with the InfraRed Spectrograph instrument (IRS; Houck et al. 2004) in spectral mapping mode. We used the short and long wavelength (SL, 5.2-14.5 $\mu \mathrm{m}$; LL, $14.0-38.0 \mu \mathrm{m})$ low resolution modules $(R \sim 60-130)$.

Data cubes were built using CUBISM (Smith et al. 2007). We performed background subtraction by using off-source data that were taken simultaneously with on-source data (see Fig. 1). These background samples were chosen by comparing the spectra of different regions throughout the field, and by checking for non-negative pixels in order to isolate the mid-IR emission coming from IC 63 and IC 59. The background samples were combined using the Average + Min-Max Trim option. Differences between the resulting sky spectra using the different background datasets are $\lesssim 0.5 \mathrm{MJy} \mathrm{sr}^{-1}$. Edge effects were also removed using CUBISM. The rest of bad pixels were removed using the automated-bad-pixel routine, as well as by interactive inspection of the spectra.

Once the data cubes were built, they were aligned spatially and resampled to the SL scale $\left(1.8^{\prime \prime} /\right.$ pixel). We retrieved the $1 \mathrm{D}$ spectra of each pixel from each data cube. Consecutive orders were averaged in the regions of overlap, which is $7.5-7.6 \mu \mathrm{m}$ between SL2 and SL1 orders, 14.2-14.7 $\mu \mathrm{m}$ between SL1 and LL2 orders, and 20.5-21.0 $\mu \mathrm{m}$ between LL2 and LL1 orders. No scaling was necessary after performing the background subtraction. The spectra were finally re-binned in a $2 \times 2$ pixel step.

In order to derive $\mathrm{PAH}$ band ratios, we decomposed the spectra using spline functions to distinguish the emission from emission lines (e.g., [Ne II], $\mathrm{H}_{2}$ ) and PAH bands, with respect to the emission from the underlying plateaus at 5-10, 10-15, and 15-18 $\mu \mathrm{m}$ (e.g., Boersma et al. 2014; Peeters et al. 2017). The specifications of the method have been described in Andrews et al. (2015). Here, we summarize it as follows: first we remove the continuum underneath the plateaus and PAH bands by 


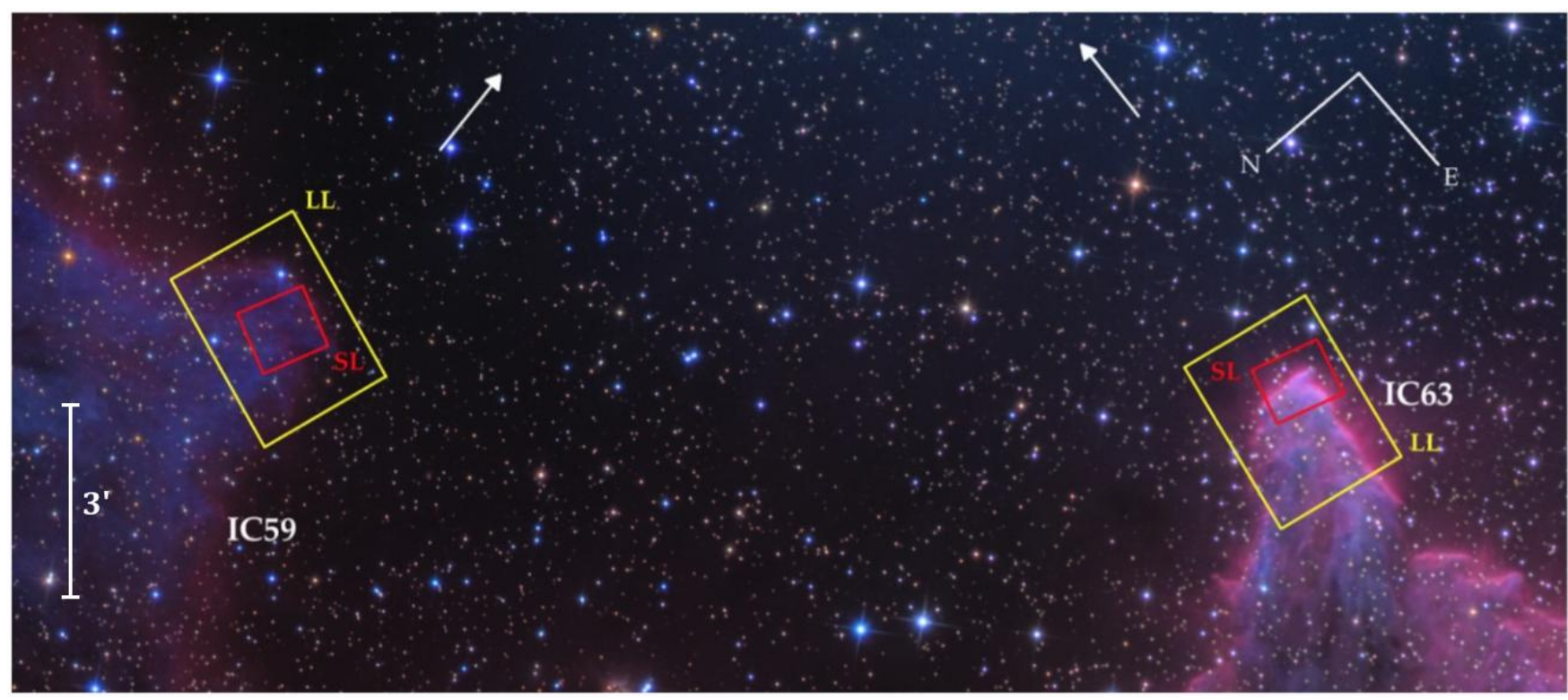

Fig. 1. Spitzer IRS data at the tip of both IC 63 and IC 59. SL datasets are shown in red rectangles, and LL datasets are depicted in yellow. IC 59 data were acquired on the same day, and with the same instrument and settings as the IC 63 data. The background samples were chosen by checking for non-negative pixels in the background-subtracted spectra of different regions in IC 63 as well as in IC 59 in order to isolate the mid-IR emission coming from both nebulae. The image of both nebulae has been taken from the Astronomy Picture of the Day archive (http://apod.nasa.gov/apod/ap111103.html; image credit Ken Crawford). The white arrows point toward the star $\gamma$ Cas, which is about 20 arcmin from the tip of IC 63.

fitting a spline function, using as anchor points the local minima at $5.5,10.3,10.5,15.0$, and $19.6 \mu \mathrm{m}$. After this, we distinguish the emission from the broad plateaus from that of the narrower PAH bands and emission lines, by using anchor points at around 5.5, 6.6, 7.2, 8.1, 8.4, 8.8, 9.4, 9.9, and $10.3 \mu \mathrm{m}$ for the features rising above the first plateau between $5-10 \mu \mathrm{m}$; at $10.3,10.5$, $11.8,12.2,13.2,14.5$, and $15.0 \mu \mathrm{m}$ for the features of the second plateau at 10-15 $\mu \mathrm{m}$; and at 15.0, 15.6, 16.2, 16.7, 17.5, 18.0, 19.4 , and $19.6 \mu \mathrm{m}$ for the third plateau at $15-20 \mu \mathrm{m}$. These local anchor points proved to properly isolate the PAH bands. The integrated intensities were obtained by fitting multi-Gaussian profiles to the remaining flux using the least square curve fitting routine MPFITFUN in IDL (Markwardt 2009). Finally, the narrow emission lines were subtracted, in order to isolate the emission coming from the PAH features and plateaus.

We also analyzed the spectra using the PAHFIT routine from Smith et al. (2007), which fits the spectra assuming the plateaus are an inherent part of the PAH bands (Sellgren et al. 2007; Smith et al. 2007). We did this to check the correlations between the observed PAH band ratios, as general trends have been found to be the same irrespective of the decomposition method used (Galliano et al. 2008). In the following we only present the spline decomposition results, unless stated otherwise.

\subsection{Herschel}

\subsubsection{PACS photometry}

The datasets were downloaded from the Herschel science archive (HSA). We retrieved data taken with the photodetector array camera and spectrometer (PACS) at 70 and $160 \mu \mathrm{m}$ (Poglitsch et al. 2010), and the Spectral and Photometric Imaging REceiver (SPIRE) at 250, 350, and $500 \mu \mathrm{m}$ (Griffin et al. 2010). The photometric data was reduced with the Herschel interactive processing environment (HIPE) version 14.0 with the recommended pipeline for the reduction of observations of extended sources. The PACS data was obtained in scan map observation mode, and it was treated with the MADmap pipeline (Cantalupo et al. 2010). The SPIRE maps were obtained with the Naive Scan Mapper task. We considered flux calibration errors to be of $15 \%$ for PACS and 7\% for SPIRE data (Griffin et al. 2010; Swinyard et al. 2010). The PSF FWHM is $5.2^{\prime \prime}$ and $12^{\prime \prime}$ for the 70 and $160 \mu \mathrm{m}$ images, while for the SPIRE data is $18^{\prime \prime}, 25^{\prime \prime}$, and $37^{\prime \prime}$ at 250,350 , and $500 \mu \mathrm{m}$, respectively.

\subsubsection{PACS line spectroscopy}

For both nebulae, spectroscopic observations of the [C II] $157 \mu \mathrm{m}$ and [OI] $63 \mu \mathrm{m}$ cooling lines were obtained with PACS and retrieved from the HSA. The observations are centered on the tip of each nebulae, coinciding with the IRS SL fields (see Fig. 1). The observations were carried out using the unchopped spectroscopy mode. Background observations were also obtained. The data was reduced with the unchopped line scan pipeline for transient correction (Fadda et al. 2016). The beam FWHM varies from about 9 " to $13^{\prime \prime}$ between the spectral range covered by PACS (55 and $180 \mu \mathrm{m})$. The spectral resolution at 63 and $157 \mu \mathrm{m}$ correspond to $R \sim 3400$ and 1500 , respectively. The line fluxes were extracted by fitting a local continua and a Gaussian line profile to the data. Intensity maps of the integrated flux were then created using HIPE. The final integrated maps are about $120^{\prime \prime} \times 120^{\prime \prime}$ centered on the tips of each nebulae.

\subsection{SOFIA velocity maps}

We obtained velocity maps of the [C II] $157 \mu \mathrm{m}$ line for IC 59 with the German REceiver for Astronomy at Terahertz frequencies (GREAT) spectrometer on board of SOFIA (Heyminck et al. 2012; Young et al. 2012; Temi et al. 2014). The observations were performed in two runs: one in May 2014 and the other in January 2015 (program ID: 02_0090, PI: E. Peeters). The observations were carried out in on-the-fly mapping mode, in combination with position switching mode. Data reduction was done with the CLASS software (Pety 2005), and the spatial resampling and 

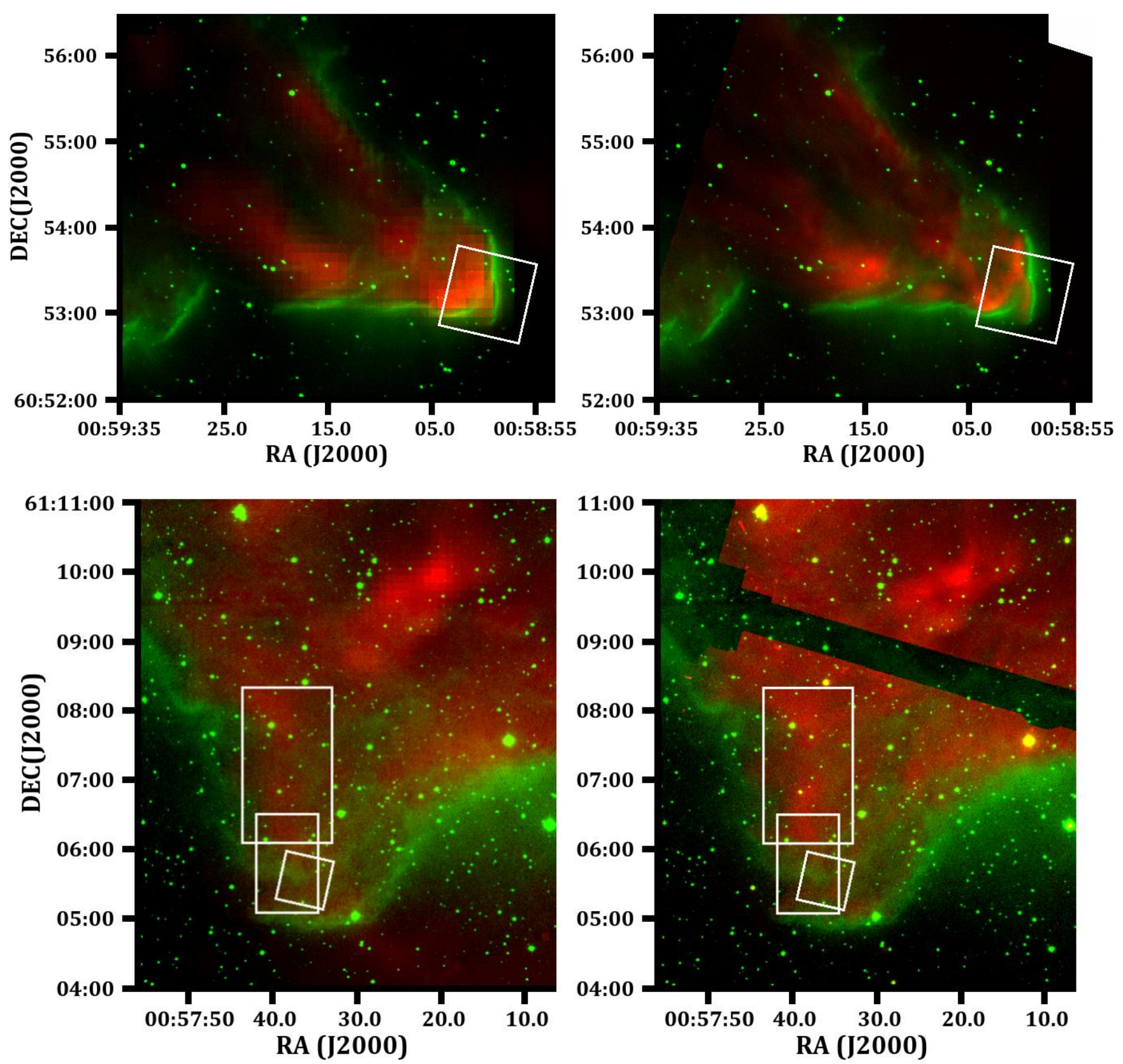

Fig. 2. Two-color images of IC 63 (top panels) and IC 59 (bottom panels). The figures show a comparison between the H $\alpha$ emission (in green) from the INT-WFC Photometric H $\alpha$ Survey of the northern Galactic plane (IPHAS; Drew et al. 2005; Barentsen et al. 2014), and the IRAC $8 \mu \mathrm{m}$ image taken with Spitzer (in red, right column), and the PACS $160 \mu \mathrm{m}$ image taken with Herschel (in red, left column). The regions covered by IRS spectral data are shown for both nebulae. For IC 59 we have also added the fields observed with SOFIA in the two runs (white rectangles aligned parallel to the declination axis).

further analysis was done with IDL. The final field of the May 2014 run is about $65^{\prime \prime} \times 110^{\prime \prime}$ at the tip of the nebula, while that of the January 2015 run is about $75^{\prime \prime} \times 130^{\prime \prime}$, northeast of the tip (see Fig. 2). The spectra were resampled to $0.12 \mathrm{~km} \mathrm{~s}^{-1}$ resolution for the May 2014 run, and $0.48 \mathrm{~km} \mathrm{~s}^{-1}$ for the January 2015 run.

\section{Results}

\section{1. $\mathrm{PAH}$ emission}

Figure 3 shows the average mid-IR spectra of the tip of the nebulae. For IC 63 we have considered the region where the PAH emission is the brightest (white square in Fig. 4). For IC 59 on the other hand, we have considered the average of the entire SL-IRS field (see Fig. 2), as these spectra have low S/N.

In general, both spectra are similar in shape but differ greatly in intensity, as the emission from the tip of IC 63 is brighter than that of IC 59 (e.g., the intensity at $11.3 \mu \mathrm{m}$ in IC 63 spectrum is $\sim 130 \mathrm{MJy} \mathrm{sr}^{-1}$, while in IC 59 spectrum is $\sim 9 \mathrm{MJy} \mathrm{sr}^{-1}$ for the applied apertures). Both spectra present the same PAH bands, as well as $\mathrm{H}_{2}$ lines and the [Ne II] $12.8 \mu \mathrm{m}$ ionization line (the [S III] $18.7 \mu \mathrm{m}$ line can be seen in some spectra in IC 63 but is not discernible in IC 59). Also, both spectra present similar $\mathrm{PAH}$ band ratios (see Table 1). No $\mathrm{C}_{60}$ emission was detected at $18.9 \mu \mathrm{m}$. 

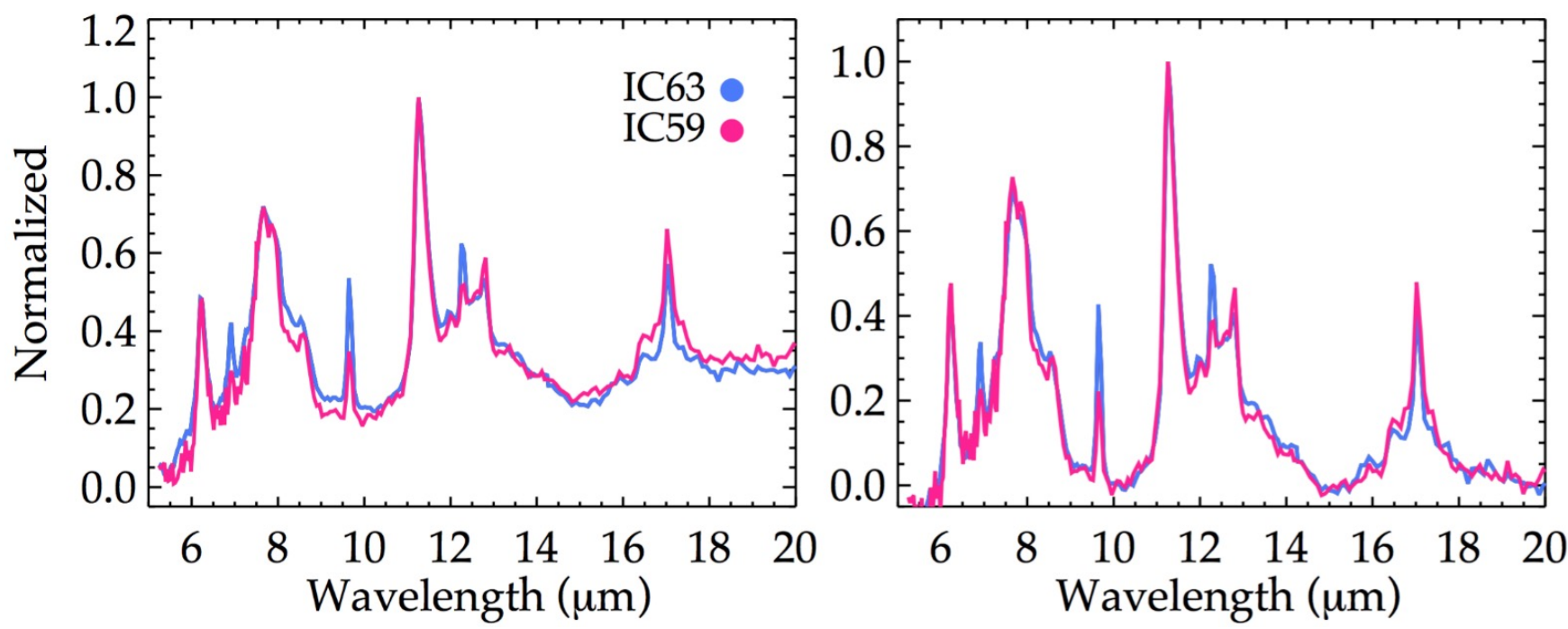

Fig. 3. Mid-IR spectra of IC 63 and IC 59 observed with the IRS instrument on board Spitzer. The spectra correspond to the SL and LL modules. For IC 63 , we have chosen a $15^{\prime \prime} \times 15^{\prime \prime}$ region at the tip of the nebula, along the second front seen in Fig. 4 where the PAH emission is the brightest. However, inspection of the entire data cube shows only small variations in the PAH band ratios throughout the tip of the nebula ( $\$ 40 \%)$. For IC 59, we took the average spectrum over a $25^{\prime \prime} \times 25^{\prime \prime}$ region at the tip of the nebula (see Fig. 2). Left panel: both spectra normalized to the peak intensity of the $11.3 \mu \mathrm{m}$ feature (132.14 $\mathrm{MJy} \mathrm{sr}^{-1}$ for IC 63 and $9.34 \mathrm{MJy} \mathrm{s}^{-1}$ for IC 59). Right panel: spectra normalized to the peak intensity of the $11.3 \mu \mathrm{m}$ feature, after the subtraction of the continuum between 5 and $20 \mu \mathrm{m}$.
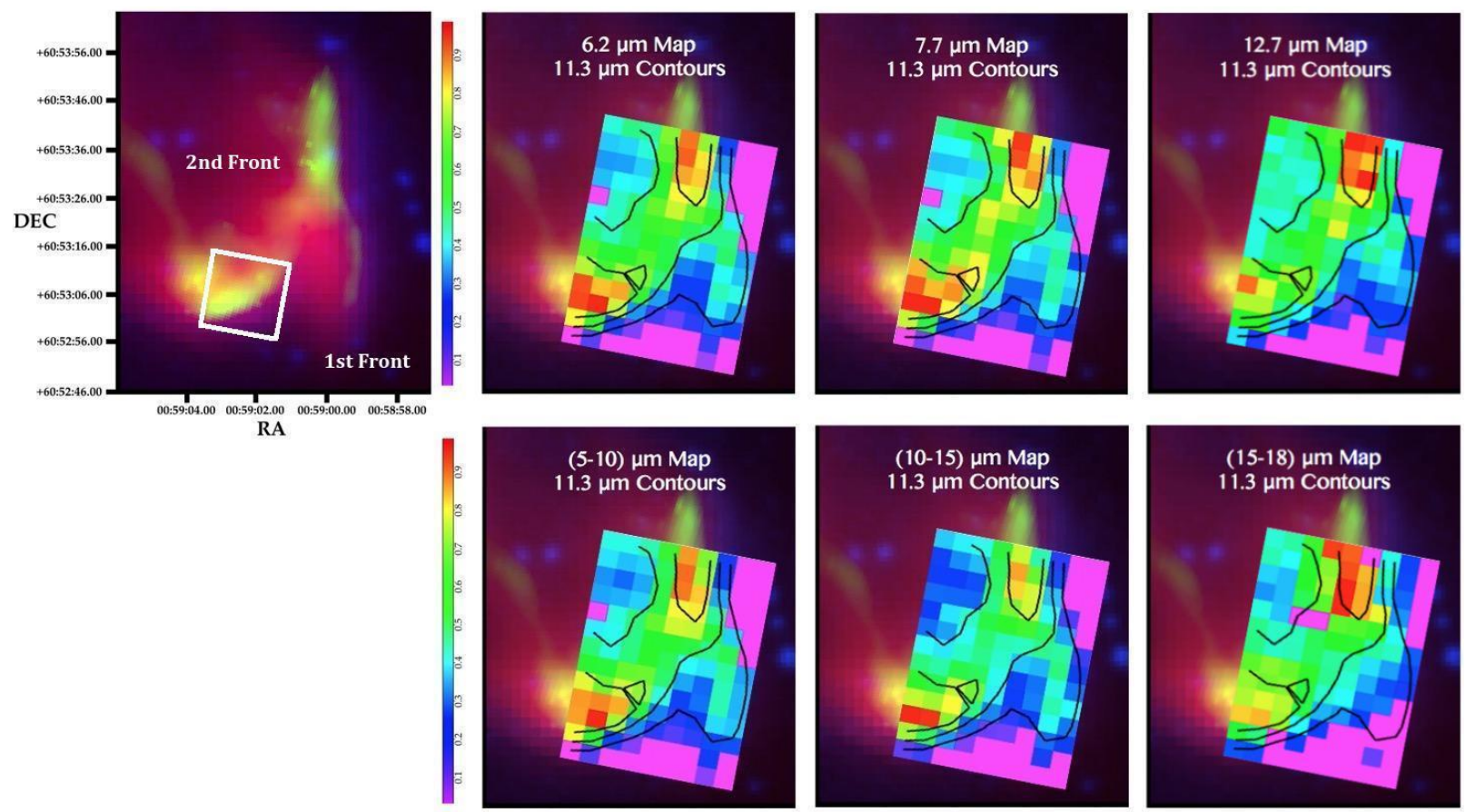

Fig. 4. Surface brightness maps of PAH emission at the tip of IC 63. The maps are $13 \times 9$ pixels (3.6"/pixel) in size. They have been overlayed on top of a three-color image made from DSS (blue), IRAC $8.0 \mu \mathrm{m}$ (green) and PACS $160 \mu \mathrm{m}$ images (red). The star $\gamma$ Cas is located $\sim 20$ arcmin to the southwest of the tip (see also Fig. 1). The maps have been normalized to their maximum peak value, and we have overplotted the contours of the $11.3 \mu \mathrm{m}$ band spatial distribution for comparison. The white square in the first map indicates the region (along the second front) where the PAH emission is the brightest, and whose average spectrum is shown in Fig. 3.

Looking at the spatial distribution of PAH emission at the tip of IC 63 (Fig. 4), we can distinguish a first front sticking out in the western part of the cloud (closest in projection to the star), and a second one with two bright regions where the PAH emission is more intense. The spatial distribution of the 6.2, 7.7, 11.3, and $12.7 \mu \mathrm{m}$ bands follow each other, as well as the spatial distribution of the 5-10 and $10-15 \mu \mathrm{m}$ plateaus. In contrast, the spatial distribution of the $15-18 \mu \mathrm{m}$ plateau is different from what is observed from the other PAH bands (e.g., the first front is not as clear as in the other bands). In the case of IC 59, the poor signal of the spectra does not allow an analysis of the spatial distribution of PAH emission on a pixel-to-pixel basis.

From Fig. 5 we find that contrary to most studies in the literature (e.g., Hony et al. 2001; Peeters et al. 2002), IC 63 does not show a clear correlation between the 6.2/11.3 and 7.7/11.3. This correlation has been linked to the ionization conditions 

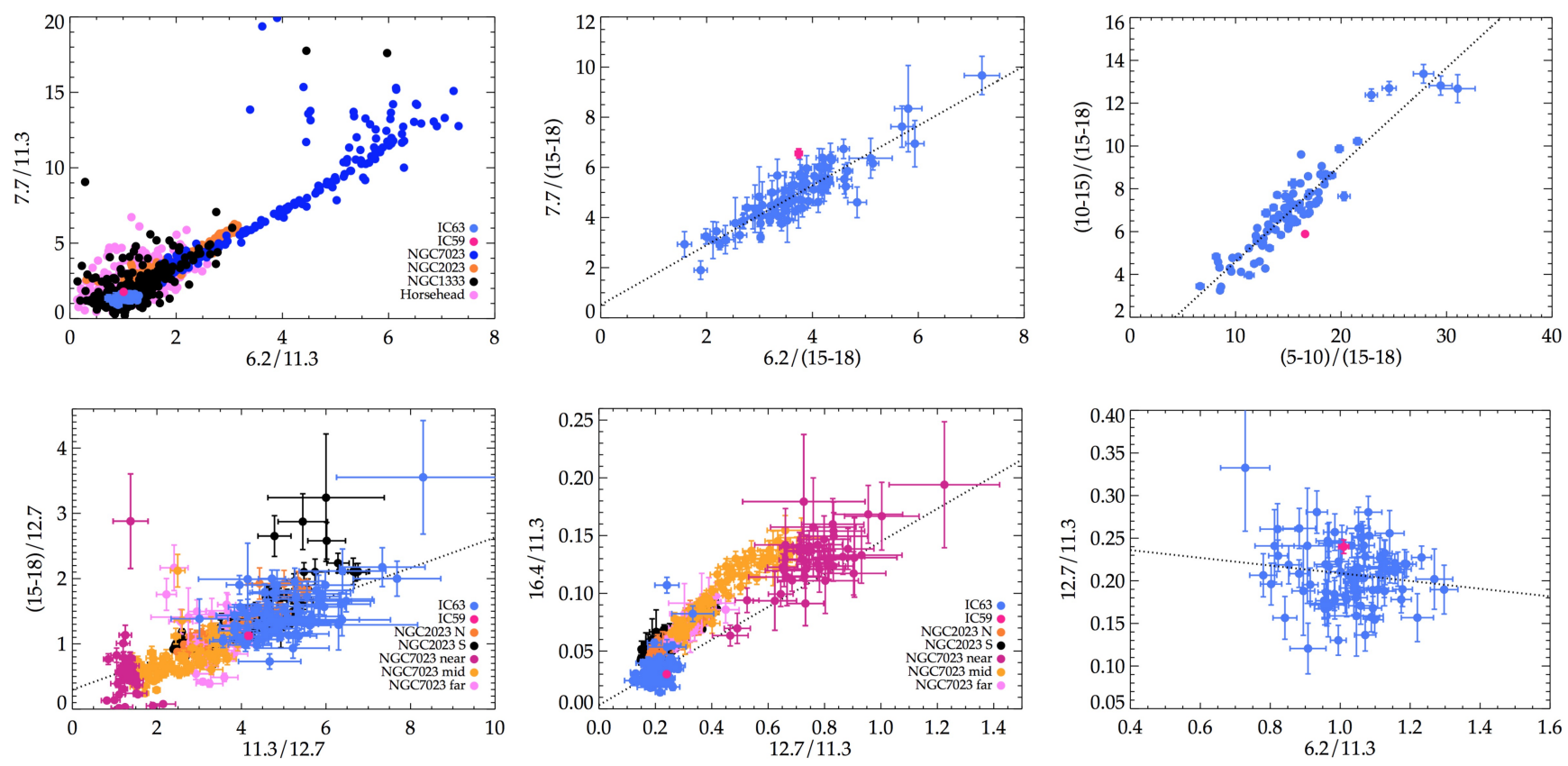

Fig. 5. Correlations between PAH band ratios observed at the tip of IC 63. We also show the PAH band ratios obtained from the average spectrum of IC 59 shown in Fig. 3 (plotted in the same color as in Fig. 3). We have plotted the data published in Stock et al. (2016, Spitzer-IRS SL data from $5-15 \mu \mathrm{m}$ ) in the 6.2/11.3 versus 7.7/11.3 correlation plot. We note that the ratios published in their work are obtained using different anchor points than ours. However, for building this plot, we have plotted their data derived from a spectral decomposition consistent with our method. The data of NGC 2023 north (N) and south (S) regions, as well as the data for NGC 7023 ("near", "mid" and "far") have been taken from Shannon et al. (2015, Spitzer-IRS SH data from 10-20 $\mu \mathrm{m}$ ). The different regions in NGC 7023 are at different distances from the star and trace the cavity (near region), the PDR front (mid region), and the cloud (far region, as defined by Shannon et al. 2015). The dotted lines correspond to the linear fits derived from the data points (see also Table 2).

Table 1. PAH band ratios at the tip of IC 63 and IC 59.

\begin{tabular}{cccccc}
\hline \hline & \multicolumn{2}{c}{ Spline } & & \multicolumn{2}{c}{ PAHFIT } \\
\cline { 2 - 3 } \cline { 5 - 6 } Ratio & IC 63 & IC 59 & & IC 63 & IC 59 \\
\hline $6.2 / 11.3$ & $1.04 \pm 0.008$ & $1.01 \pm 0.014$ & & $0.94 \pm 0.007$ & $0.93 \pm 0.013$ \\
$7.7 / 11.3$ & $1.39 \pm 0.055$ & $1.77 \pm 0.046$ & & $1.86 \pm 0.021$ & $2.20 \pm 0.040$ \\
$8.6 / 11.3$ & $0.18 \pm 0.005$ & $0.22 \pm 0.007$ & & $0.35 \pm 0.005$ & $0.35 \pm 0.008$ \\
$12.7 / 11.3$ & $0.18 \pm 0.006$ & $0.24 \pm 0.008$ & & $0.48 \pm 0.004$ & $0.49 \pm 0.006$ \\
$16.4 / 11.3$ & $0.02 \pm 0.001$ & $0.03 \pm 0.001$ & & $0.03 \pm 0.001$ & $0.02 \pm 0.001$ \\
$(5-10) / 11.3$ & $4.41 \pm 0.029$ & $4.48 \pm 0.050$ & & $\ldots$ & $\ldots$ \\
$(10-15) / 11.3$ & $2.09 \pm 0.013$ & $1.59 \pm 0.016$ & & $\ldots$ & $\ldots$ \\
$(15-18) / 11.3$ & $0.28 \pm 0.002$ & $0.27 \pm 0.003$ & & $\ldots$ & $\ldots$ \\
\hline
\end{tabular}

Table 2. Correlation coefficients for IC 63 PAH band ratio plots shown in Fig. 5.

\begin{tabular}{cccc}
\hline \hline$x$-ratio vs $y$-ratio & $A$ & $B$ & Corr. Coef. \\
\hline $6.2 / 11.3$ vs. $7.7 / 11.3$ & 0.491 & 0.868 & 0.41 \\
$6.2 / 12.7$ vs. $7.7 / 12.7$ & 1.065 & 1.323 & 0.86 \\
$6.2 /(15-18)$ vs. $7.7 /(15-18)$ & 1.191 & 0.520 & 0.91 \\
$(5-10) /(15-18)$ vs. $(10-15) /(15-18)$ & 0.453 & 0.070 & 0.94 \\
$11.3 / 12.7$ vs. $(15-18) / 12.7$ & 0.233 & 0.298 & 0.57 \\
$12.7 / 11.3$ vs. $16.4 / 11.3$ & 0.142 & 0.003 & 0.40 \\
$6.2 / 11.3$ vs. $12.7 / 11.3$ & -0.045 & 0.254 & -0.14 \\
\hline
\end{tabular}

Notes. The $A$ and $B$ coefficients correspond to the parameters derived from a linear fit of the form $y=A \times x+B$. The correlation coefficients correspond to the weighted Pearson correlation coefficients between $x$ and $y$ data points. under which PAHs emit, as C-C modes are intrinsically stronger in cationic species, while $\mathrm{C}-\mathrm{H}$ modes are stronger in neutral species (e.g., Langhoff et al. 1996; Allamandola et al. 1999; Bauschlicher et al. 2008; Galliano et al. 2008). We claim the correlation cannot be observed because these ratios remain relatively constant throughout the studied region. Indeed, comparing all the available spectra at the tip of IC 63 (with enough S/N) normalized to the peak of the $11.3 \mu \mathrm{m}$ band, we get that the spectra vary $<40 \%$ throughout the studied region. Thus, we find that the PAH emission at the tip of IC 63 is remarkably steady, and overall it also agrees with the average PAH emission observed at the tip of IC 59.

Even though we do not see a correlation between the 6.2 and $7.7 \mu \mathrm{m}$ bands when normalizing by the $11.3 \mu \mathrm{m}$ band, we do see a tentative correlation when normalizing by the $15-18 \mu \mathrm{m}$ plateau $(P=0.91)$. The origin of this plateau is still under debate. It has been proposed to originate from PAH clusters and very large PAHs containing more than 200 carbon atoms (Allamandola et al. 1999; Peeters et al. 2012, 2017). The fact that we do find a correlation when normalizing by the $15-18 \mu \mathrm{m}$ plateau might be pointing toward a different origin of this plateau compared to the 6.2 and $7.7 \mu \mathrm{m}$ bands, associated with PAH species. This is also pointed out when comparing the strength of the plateaus. The only correlation we see is between the $(5-10) /(15-18)$ and the $(10-15) /(15-18)$, showing that at the tip of IC 63 , the behavior of the emission of the (15-18) plateau seems to be unique and distinct from to the shorter $(\lambda<15 \mu \mathrm{m})$ wavelength features.

Compared to PAH band ratios of other nebulae such as NGC 2023 and NGC 7023, it is clear that IC 63 (and IC 59) PAH ratios span only a small range in values (see Fig. 5), which is the origin of the lack of correlations seen in the PAH band ratios. While IC 63 does not exhibit a clear correlation between the 
6.2 and $7.7 \mu \mathrm{m}$ PAH bands, the observed ratios are consistent with the trend seen in other sources. Also, Shannon et al. (2015) found a correlation between the 11.3/12.7 and the (15-18)/12.7. Even though we do not see this correlation in our data alone $(P=0.57$; see Fig. 5), the data points of IC 63 (and IC 59) follow the correlation found for NGC 2023 and NGC 7023. In addition, three different regions are indicated for NGC 7023 at different distances from the star, and trace the cavity ("near" region), the PDR front ("mid" region), and the cloud ("far" region; see Shannon et al. 2015 for details). As can be seen in the 11.3/12.7 and the (15-18)/12.7 correlation plot, these three regions occupy different sectors in the plot: the PAH ratios of the near region have the smallest ratios, the mid region (where the PAH emission is the brightest) has intermediate values, and the PAH ratios from the "far" region (the more shielded region) are the largest ones. When compared to IC 63 (and IC 59) PAH ratios, we see these are more extreme than those from the far region in NGC 7023 (i.e., are at higher 11.3/12.7 and (15-18)/12.7).

This sort of sequence is also observed for the correlation between the 12.7/11.3 and 16.4/11.3 shown in Fig. 5. Also, visual comparison with the data published in Boersma et al. (2014) for the north-western PDR of NGC 7023 shows a similar trend in the 6.2/11.3 versus $12.7 / 11.3$ plot. Comparing IC 63 (IC 59) PAH band ratios to those derived for NGC 2023 or NGC 7023, we notice again that our ratios do not vary as much as for the other objects (Boersma et al. 2014; Shannon et al. 2015). For the $12.7 / 11.3$ versus $16.4 / 11.3$ correlation plot, we also notice the same sequence: the smaller ratios for NGC 7023 are from regions farther away from the star, with IC 63 (and IC 59) exhibiting the smallest PAH band ratios. This suggests that the population of PAHs emitting in IC 63 (and IC 59) are most likely less ionized than the emitting PAHs in the more shielded parts of NGC 7023 and NGC 2023.

\subsection{Physical conditions}

\subsubsection{Dust temperature}

From the PACS 70 and $160 \mu \mathrm{m}$ images we determined color dust temperatures, assuming a modified blackbody for the dust with a spectral emissivity index $\beta=1.8$ (Dupac et al. 2003). Figure 6 shows the dust temperature maps for both nebulae, compared to the $\mathrm{H} \alpha$ images from IPHAS. The dust temperatures span similar ranges between both objects. Considering other $\beta$ values between 1 and 2 does not change the spatial distributions, nor the derived values significantly $\left(\Delta T_{\text {dust }} \sim 2 \mathrm{~K}\right)$. Overall for IC 63, the dust temperature distribution is rather homogeneous: at the position of the spectrum shown in Fig. 3 the dust temperature is about $30 \mathrm{~K}$, similar to the value derived for the region centered on position $(\mathrm{RA}, \mathrm{Dec})=(00: 59: 15,+60: 53: 15)$ which appears to be another region where the PAH emission is as bright as in the tip (see Fig. 2).

In the case of IC 59, the region covered by the IRS-SL data (the tip) is not where the radiation of the star is directly heating the dust. This appears to happen instead at the western side of the cloud, in the concave part at around position (RA, Dec) = $(00: 57: 15,+61: 06: 00)$. This region is in fact in the radial direction to the star (see white arrow in Fig. 1). The temperature there is about $29.5 \mathrm{~K}$, while at the tip of the nebula the temperature is slightly lower, $T_{\text {dust }} \sim 27 \mathrm{~K}$.

\subsubsection{UV radiation field incident on IC $63 /$ IC 59}

We derive the intensity of the UV radiation field $G_{0}$ at the tip of IC 63 and IC 59 directly from integrating the spectral energy distributions (SEDs). The SEDs of IC 63 and IC 59 were built from convolving all photometric images to match the SPIRE $500 \mu \mathrm{m}$ image resolution, using the kernels of Gordon et al. $(2008)^{1}$ for each specific filter: IRAC $3.6,4.5,5.8$, and $8.0 \mu \mathrm{m}$, MIPS $24 \mu \mathrm{m}$, PACS 70 and $160 \mu \mathrm{m}$, and SPIRE 250, 350, and $500 \mu \mathrm{m}$ (see Table 3). Assuming a spectral emissivity index $\beta=$ 1.8, we fit the far IR spectral region of the SEDs with a onecomponent modified blackbody model where the flux density is described as $S_{\lambda} \propto \lambda^{-\beta} B_{\lambda}\left(T_{\text {dust }}\right)$ leaving as free parameters the dust opacity at $160 \mu \mathrm{m}$ and the dust temperature $T_{\text {dust }}$. The results of the fit lead to opacities and dust temperatures of $\tau_{160}=8 \times 10^{-4}$ and $T_{\text {dust }} \sim 30 \mathrm{~K}$ for IC 63, and $\tau_{160}=2 \times 10^{-4}$ and $T_{\text {dust }}=27 \mathrm{~K}$ for IC 59. The resulting intensities of the UV field were estimated following Meixner et al. (1992). Assuming that all the UV radiation incident on the tip of the nebulae is reemitted and observable at IR wavelengths, and considering the geometrical factor equal to one (assumed for spherical geometries) we obtain a $G_{0} \sim 150$ at the tip of IC 63 , and a $G_{0} \sim 25$ at the tip of IC 59.

The values obtained are several times lower than what has been adopted in previous studies. Most studies on IC 63 have assumed an incident UV radiation field of 650 Draine field units at the tip of IC 63 based on the work of Jansen et al. (1994). In that study they derived the emission from $\gamma$ Cas based on spectrophotometric measurements (Code \& Meade 1979) and broad band flux at $950 \AA$ (Troy et al. 1975). According to this, they estimated an extinction corrected flux at $1000 \AA$ of $F_{\text {IC63 }}=2.2 \times 10^{-5}$ photons s${ }^{-1} \mathrm{~cm}^{-2} \mathrm{~Hz}^{-1}$, assuming the projected distance between IC 63 and the star $(1.3 \mathrm{pc})$ as the actual distance between the sources. This is equivalent to $\sim 650$ Draine field units, 1100 Habing field units and 900 Mathis field units at $1000 \AA$. Later on, France et al. (2005) directly measured the UV emission of $\gamma$ Cas using a flux calibrated FUSE spectrum. Assuming again the projected distance of $1.3 \mathrm{pc}$ as the actual distance between the star and the nebula, these authors found that, the UV radiation field incident on IC 63 is a bit lower than what Jansen et al. (1994) predicted: assuming the distance from Earth to $\gamma$ Cas is $230 \mathrm{pc}$ (Vakili et al. 1984) then the UV radiation incident on IC 63 is $F_{\text {IC63 }}=1.9 \times 10^{-5}$ photons s$~^{-1} \mathrm{~cm}^{-2} \mathrm{~Hz}^{-1}$ at $1000 \AA$, which at $d_{\text {IC63 }}=1.3$ pc is equivalent to 950 Habing field units approximately.

We acknowledge that the estimates derived from the SEDs are strongly subjected to the geometry assumed for each system. In contrast with previous studies, our derived values would imply that IC 63 is at least three times farther away than the projected distance to the star. Similarly, the tip of IC 59 is even farther away than IC 63, at least five times its projected distance to $\gamma$ Cas $(1.5 \mathrm{pc})$. Just recently, studies on the polarization of light through IC 63 and IC 59 have suggested this as a possible scenario (Andersson et al. 2013; Soam et al. 2017). Analyzing a narrow band emission image of the $2.12 \mu \mathrm{m} \mathrm{H}_{2}$ line, Andersson et al. (2013) found "streamers" that are oriented at a steeper position angle than the projected radius between the star and IC 63 . Based on this, they estimated an actual distance of $2 \mathrm{pc}$ instead of 1.3 pc. Soam et al. (2017) on their study of the magnetic field in IC 63 and IC 59 observed that the radiation from $\gamma$ Cas hits the concave part of the M shape of IC 59 (around position (RA, Dec $)=(00: 57: 15,+61: 06: 00)$ in Fig. 2), and therefore the tip of IC 59 (where IRS SL data was taken) is probably farther away from the star than the concave part (see Fig. 2).

1 http://dirty.as.arizona.edu/ kgordon/mips/conv_psfs/ conv_psfs.html 

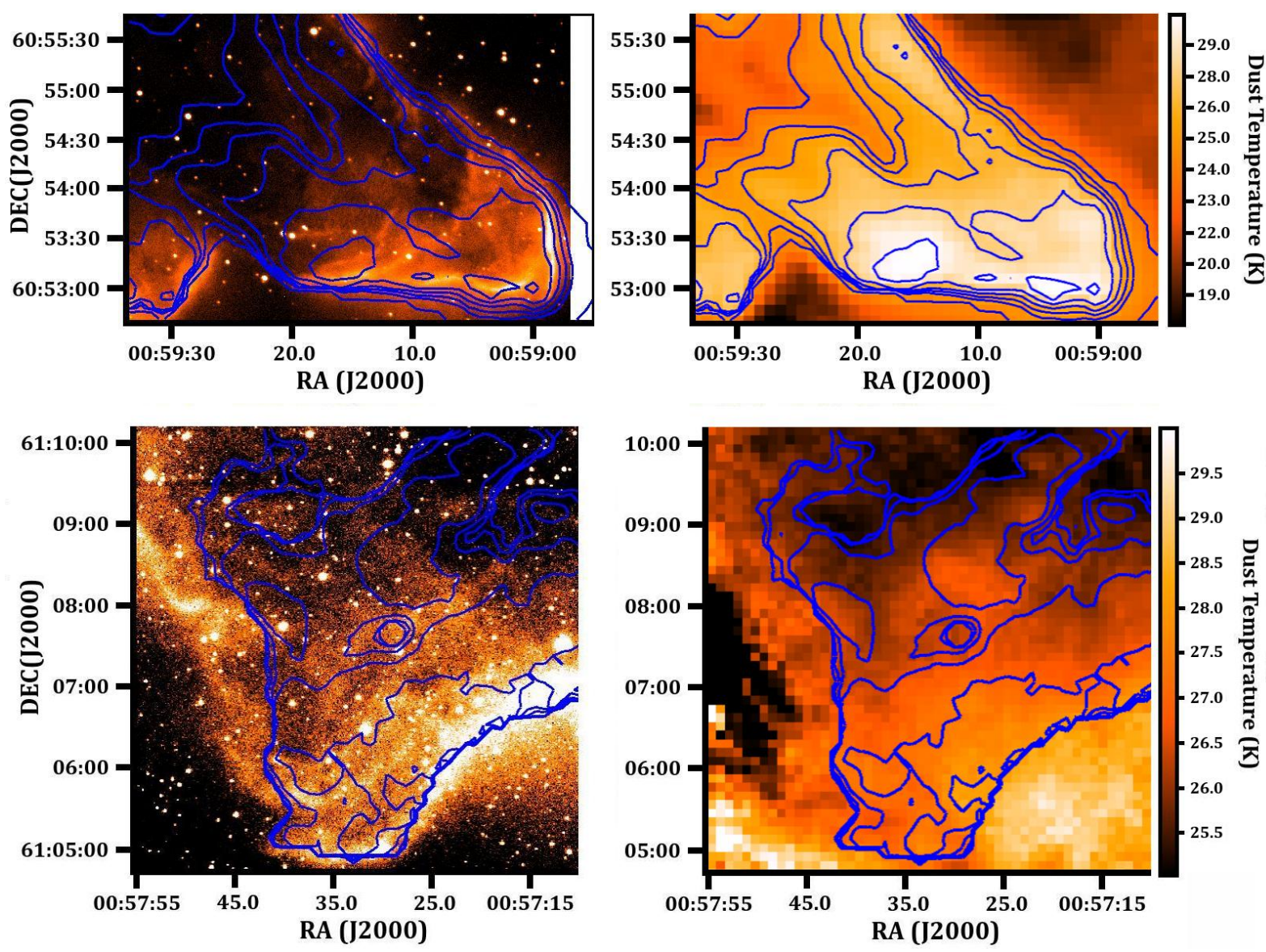

Fig. 6. Dust temperature maps (right panels) and its comparison to the $\mathrm{H} \alpha$ emission from IPHAS (left panels) for both, IC 63 (top panels) and IC 59 (bottom panels). The blue contours correspond to the contours of the dust temperature maps considering intensities above a $4 \sigma$ cut. We have plotted these contours for visual guidance, as they delineate well the emission at 70 and $160 \mu \mathrm{m}$.

Figure 7 shows a direct comparison of the SEDs of IC 63 and IC 59 with those of other well-known objects such as the Horsehead PDR and NGC 7023 taken from the literature (Köhler et al. 2014; Ochsendorf \& Tielens 2015). Compared to other PDRs we see that IC 63 and IC 59 have SEDs similar to those of regions of moderate-to-low $G_{0}$ values, such as NGC $7023 \mathrm{E}\left(G_{0}=250\right.$, $T_{\text {dust }}=20 \mathrm{~K}$; Pilleri et al. 2012; Köhler et al. 2014) and the Horsehead PDR $\left(G_{0}=100, T_{\text {dust }}=23 \mathrm{~K}\right.$; Ochsendorf \& Tielens 2015). The proto-typical and well-studied NGC 7023 N PDR on the other hand is at a higher $G_{0} \sim 2600$ (Pilleri et al. 2012; Okada et al. 2013; Köhler et al. 2014). We notice both IC 63 and NGC $7023 \mathrm{~N}$ peak at similar wavelengths $\left(\lambda_{\text {peak }} \sim 100 \mu \mathrm{m}\right.$, $\left.T_{\text {dust }} \sim 3000 / \lambda_{\text {peak }}\right)$. However, the surface brightness of IC 63 is more than an order of magnitude less than that of NGC $7023 \mathrm{~N}$. Thus, we suspect the irradiated surface is either much smaller than what we assume (i.e., the geometrical factor is much less than 1), or IC 63 is a clumpy medium with a surface filling factor less than 0.1 (and thus most of the UV radiation can escape through the cloud).

\subsubsection{Gas temperature}

We built excitation diagrams of $\mathrm{H}_{2}$ to derive the excitation temperature of the molecular hydrogen gas (see Fig. 8). We assume that the lines are optically thin, and we adopt an ortho-to-para ratio of three. The properties of each level (upper level energies and Einstein coefficients) have been taken from Rosenthal et al. (2000). We find two temperature components for both nebulae.
Table 3. Photometric intensities at the tip of IC 63 and IC 59.

\begin{tabular}{cccccc}
\hline \hline & \multicolumn{2}{c}{ IC 63 } & & \multicolumn{2}{c}{ IC 59 } \\
\cline { 2 - 3 } \cline { 5 - 6 }$\lambda(\mu \mathrm{m})$ & $I_{\nu}(\mathrm{MJy} / \mathrm{sr})$ & $\delta I_{v}$ & & $I_{v}(\mathrm{MJy} / \mathrm{sr})$ & $\delta I_{v}$ \\
\hline 3.6 & 2.8 & 0.3 & & 0.4 & 0.1 \\
4.5 & 1.2 & 0.1 & & 0.2 & 0.1 \\
5.7 & 11.2 & 1.6 & & 2.4 & 0.8 \\
7.9 & 37.3 & 3.6 & & 3.5 & 1.1 \\
23.7 & 46.0 & 5.2 & & 7.4 & 1.4 \\
70.0 & 611.0 & 6.4 & & 33.4 & 2.4 \\
160.0 & 543.3 & 5.6 & & 70.2 & 3.1 \\
250.0 & 230.7 & 5.6 & & 35.2 & 2.9 \\
350.0 & 102.9 & 4.4 & & 17.5 & 2.3 \\
500.0 & 40.6 & 4.6 & & 7.6 & 1.5 \\
\hline
\end{tabular}

For IC 63 we find the cooler component has a temperature of $T=207 \pm 30 \mathrm{~K}$ and a high column density of $2.3 \times 10^{20} \mathrm{~cm}^{-2}$. The warmer component is at $T=740 \pm 47 \mathrm{~K}$ and has a lower column density of $9.3 \times 10^{17} \mathrm{~cm}^{-2}$. For IC 59 we find slightly cooler temperatures and lower densities, although agreeing within errors to those derived for the tip of IC 63: the cooler component is at $T=150 \pm 34 \mathrm{~K}$ and has a high column density gas of $1.2 \times 10^{20} \mathrm{~cm}^{-2}$, while the low column density gas $\left(1.8 \times 10^{17} \mathrm{~cm}^{-2}\right)$ is at $T=675 \pm 49 \mathrm{~K}$. These estimates appear to be in good agreement with the values determined for IC 63 from Thi et al. (2009) using ISO data. 


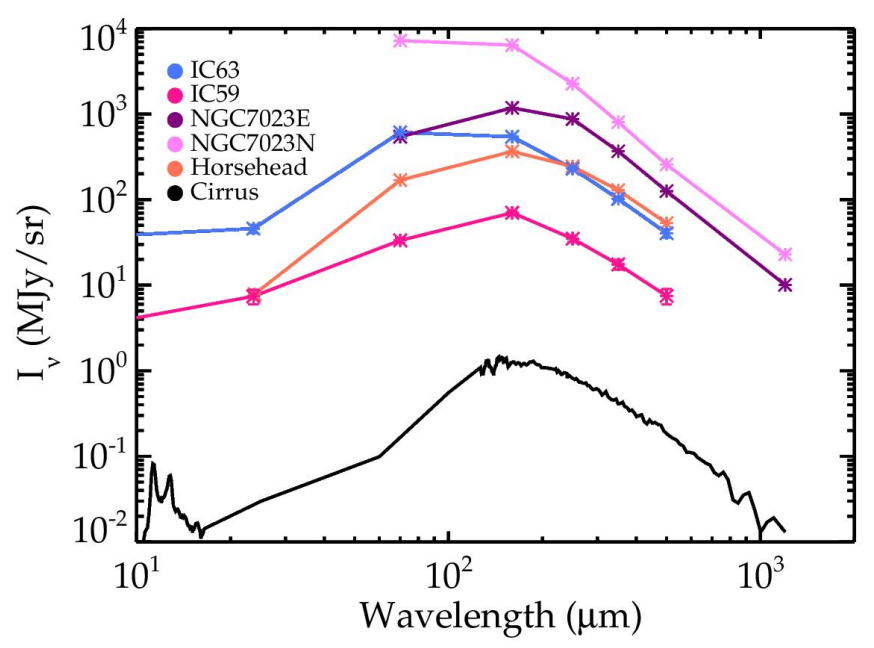

Fig. 7. Far IR spectral energy distribution of IC 63 and IC 59 compared to that of the Horsehead PDR $\left(G_{0}=100\right.$ and $T_{\text {dust }}=23.3 \mathrm{~K}$ for a spectral index $\beta=0.99$; Ochsendorf \& Tielens 2015), and NGC7023 eastern (E) and north (N) PDRs $\left(G_{0}=250\right.$ and 2600 , and $T_{\text {dust }} \sim 20$ and $30 \mathrm{~K}$, respectively assuming $\beta=2$; Köhler et al. 2014). Also shown is the SED derived for the cirrus cloud in the diffuse ISM (Compiègne et al. 2010). For IC 63 we found $G_{0}=150$ and $T_{\text {dust }} \sim 30 \mathrm{~K}$, while for IC 59 we derive values of $G_{0}=25$ and $T_{\text {dust }}=27 \mathrm{~K}$.

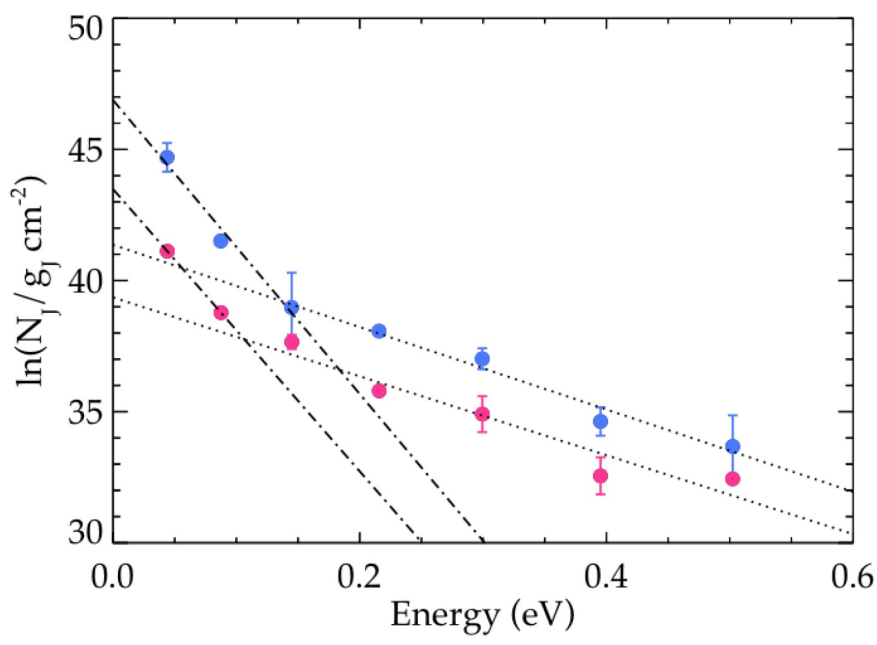

Fig. 8. $\mathrm{H}_{2}$ excitation diagrams for IC 63 (blue) and IC 59 (red). Two temperature fits have been applied for both nebulae. For IC 63 we find that the high column density gas $\left(2.3 \times 10^{20} \mathrm{~cm}^{-2}\right)$ is at $T=207 \pm$ $30 \mathrm{~K}$ (dashed lined), while the low column density gas $\left(9.3 \times 10^{17} \mathrm{~cm}^{-2}\right)$ is at $T=740 \pm 47 \mathrm{~K}$ (dotted line). For IC 59 on the other hand, we find slightly cooler temperatures: the high column density gas $(1.2 \times$ $10^{20} \mathrm{~cm}^{-2}$ ) is at $T=150 \pm 34 \mathrm{~K}$ (dashed line), while the low column density gas $\left(1.8 \times 10^{17} \mathrm{~cm}^{-2}\right)$ is at $T=675 \pm 49 \mathrm{~K}$ (dotted line).

\subsubsection{Density}

From the diagnostic diagrams of Kaufman et al. (1999, 2006) we can constrain the density of the gas based on the intensity ratios of the cooling lines. For IC 63, we find ([O I] 63/[C II]157) = $2.04 \pm 0.52$ and $([\mathrm{C} \mathrm{II}] 157+[\mathrm{O} \mathrm{I}] 63) / I_{\mathrm{FIR}}=0.016$ at the tip of the nebula (with $I_{\mathrm{FIR}}=3.6 \times 10^{-2} \mathrm{erg} \mathrm{s}^{-1} \mathrm{~cm}^{-2} \mathrm{sr}^{-1}$ considering the factor of two that takes into account that the cooling lines are optically thick, while the dust emits in all directions). These values constrain the mean density to $1.2 \times 10^{4} \mathrm{~cm}^{-3}$ assuming a $G_{0}=150$. It is important to mention that the derived ratios fall in a region of the Kaufman diagrams where the density
Table 4. Fine structure line intensities for IC 63 and IC 59.

\begin{tabular}{cccccc}
\hline \hline & \multicolumn{2}{c}{ IC 63 } & & \multicolumn{2}{c}{ IC 59 } \\
\cline { 2 - 3 } \cline { 5 - 6 } Line & $I$ & $\delta I$ & & $I$ & $\delta I$ \\
\hline [CII] 157 & $2.01 \times 10^{-7}$ & $3.22 \times 10^{-8}$ & & $6.20 \times 10^{-8}$ & $9.86 \times 10^{-9}$ \\
[OI] 63 & $4.10 \times 10^{-7}$ & $8.26 \times 10^{-8}$ & & $5.56 \times 10^{-8}$ & $2.78 \times 10^{-8}$ \\
\hline
\end{tabular}

Notes. All intensities are given in $\mathrm{W} / \mathrm{m}^{-2} \mathrm{sr}^{-1}$.

is relatively independent of $G_{0}$. For IC 59 on the other hand, we get a lower density estimate of $2 \times 10^{3} \mathrm{~cm}^{-3}$ for a $G_{0}=25$ $(([\mathrm{OI}] 63 /[\mathrm{C} \mathrm{II}] 157)=0.90 \pm 0.47$ and $([\mathrm{C} \mathrm{II}] 157+[\mathrm{OI}] 63) /$ $\left.I_{\mathrm{FIR}}=0.019, I_{\mathrm{FIR}}=6.5 \times 10^{-3} \mathrm{erg} \mathrm{s}^{-1} \mathrm{~cm}^{-2} \mathrm{sr}^{-1}\right)$.

The fact that we find a lower density for IC 59 is consistent with the results presented in Fig. 8. Indeed, from the excitation diagrams it follows that in IC 63, levels two to four $(\mathrm{S}(0)$ to $\mathrm{S}(2)$ ) are in local-thermodynamic-equilibrium (LTE), while in the excitation diagram of IC 59 we see that levels from four on arise from UV fluorescence.

\subsubsection{Predicted gas and dust temperatures}

We can now use PDR models with the deduced density and UV radiation field strength to predict the gas and dust temperatures in IC 63 and IC 59. These predicted values can then be compared with our observationally deduced values.

Based on the deduced UV radiation field, we determine the dust temperature at the PDR front (i.e. assuming $A_{\mathrm{V}}=0$ ) following the methodology outlined by Hollenbach et al. (1991) and Tielens (2005, Eqs. 9.18, 9.19). Hereby, we assume that the dust is heated by the attenuated incident UV radiation field, the $2.7 \mathrm{~K}$ microwave background and the IR radiation from the dust itself. In this way, we obtain a dust temperature of 33 and $23 \mathrm{~K}$ for IC 63 and IC 59, respectively. This is consistent with our derived dust temperatures of 30 and $27 \mathrm{~K}$, respectively, in particular since we have determined the latter solely based on the PACS 70 and $160 \mu \mathrm{m}$ observations (thus missing longer wavelength data) resulting in larger uncertainties.

Likewise, we obtain predicted surface temperatures and $\mathrm{H}_{2}$ line ratios using the Photo Dissociation Region Toolbox ${ }^{2}$ (Kaufman et al. 2006; Pound \& Wolfire 2008). Comparison of the diagnostic diagram from PDR models from Kaufman et al. (1999) using the standard model set with the density and UV radiation field strength results in gas temperatures of approximately 130 and $90 \mathrm{~K}$ for IC 63 and IC 59, respectively. The gas temperatures we determined from the $\mathrm{H}_{2}$ excitation diagram are $207 \pm 30$ and $150 \pm 34 \mathrm{~K}$, respectively, somewhat higher than the predicted values for both sources. We note that the latter are obtained based on, respectively, two and three $\mathrm{H}_{2}$ lines, $\mathrm{S}(0)$ to $\mathrm{S}(2)$, assuming an ortho-to-para ratio of 3. Similarly, comparison of the diagnostic diagram from PDR models from Kaufman et al. (2006) with the density and UV radiation field strength results in a $S(0) / S(1)$ line ratio of approximately 2.3 and 0.7 for IC 63 and IC 59, respectively. While this is roughly in agreement with the observed value for IC 63 (4.6), it is not consistent with that for IC 59 (10.8). The larger discrepancy found for the gas temperature and the $S(0) / S(1)$ compared to the dust temperature likely arises from the true ortho-to-para ratio being less than our assumed value of 3. Such ortho-to-para ratios (being different from 3 ) have been reported for PDRs in the literature

2 http://dustem.astro. umd.edu/pdrt 
(e.g., Fuente et al. 1999; Habart et al. 2003, 2011): these reported values are smaller than 3 and vary from region to region. Indeed, the ortho-to-para ratio is influenced by non-thermal processes which are not fully accounted for in the used PDR models (Bron et al. 2016).

\section{3. [C II] velocity map of IC 59}

Figure 9 shows the [C II] $157 \mu$ m emission line velocity map obtained with SOFIA. The [C II] emission is more intense in the north part of the cloud (farther away from the star in terms of projected distance). There is very little emission at the tip of the nebula, where the SL-IRS data was taken. The velocity map shows a redshifted component that crosses the map in the southeast to northwest direction (see position (RA, Dec) = (00:57:40.0, +61:06:20)). This component lies almost perpendicular to the radial direction to the star, behind the delineated region where the dust temperature increases (see Fig. 6). This component is probably related to the movement of the material in the PDR. The redshift of the line reaches velocities of up to $+0.03 \mathrm{~km} \mathrm{~s}^{-1}$, compared to the largest blueshifts observed in the northwest part of the map and at the tip (blueshifts of up $\left.-0.74 \mathrm{~km} \mathrm{~s}^{-1}\right)$. Some blueshifted lines at $-0.67 \mathrm{~km} \mathrm{~s}^{-1}$ might show a second component, but the data is not clear enough to distinguish them quantitatively. Overall the FWHM of the [C II] line appears to be rather narrow $\left(\sim 1.75 \mathrm{~km} \mathrm{~s}^{-1}\right)$, becoming broader only in the eastern and northern area of the map, where it reaches values of $3.3 \mathrm{~km} \mathrm{~s}^{-1}$ at position (RA, Dec) = (00:57:34, +61:07:53).

We also notice the [C II] $157 \mu \mathrm{m}$ emission follows the clumps observed in the IRAC 8 and MIPS $24 \mu \mathrm{m}$ images, which show where the FUV radiation is dominating the environment. From this it appears that the cometary shape of IC 59 is more of a projection effect, where the clump at the north of the map (that is farther from the star in projection) would be actually closer to the $\gamma$ Cas

\section{Discussion}

In Sect. 3.1, we found that the PAH emission is rather uniform at the tip of IC 63, and its derived PAH band ratios are similar to those found in the average spectrum of IC 59. Even though both clouds are illuminated by the same star - and hence, we expect them to be composed of similar initial interstellar material - the similarity in their emission is still puzzling, as physical conditions may vary considerably between the two studied regions.

When comparing the PAH band ratios at the tip of IC 63 (and IC 59) to those found in other reflection nebulae like NGC 7023 and NGC 2023, we find two interesting results. First, as mentioned above, PAH band ratios do not vary much within the tip of IC 63 (and IC 59). In fact, the range of PAH band ratios seen in IC 63 and IC 59 is considerably smaller than that observed in other objects, such as NGC 7023. In this source, large variations have been found throughout a region of about $45^{\prime \prime} \times 40^{\prime \prime}$, which at a distance of about $430 \mathrm{pc}$, corresponds to a projected region of $\sim 0.09 \times 0.08 \mathrm{pc}^{2}$ (Boersma et al. 2014). The region at the tip of IC 63 on the other hand, correspond to a projected area of about $0.05 \times 0.03 \mathrm{pc}^{2}\left(47^{\prime \prime} \times 32^{\prime \prime}\right)$, assuming that IC 63 is at the same distance from Earth as $\gamma$ Cas $(\sim 230 \mathrm{pc})$.

The second interesting result is that, taking under consideration the differences between our spectral decomposition methods, IC 63 PAH band ratios (and IC 59) are close to those from "zone 5" of NGC 7023 in Boersma et al. (2014), as inferred
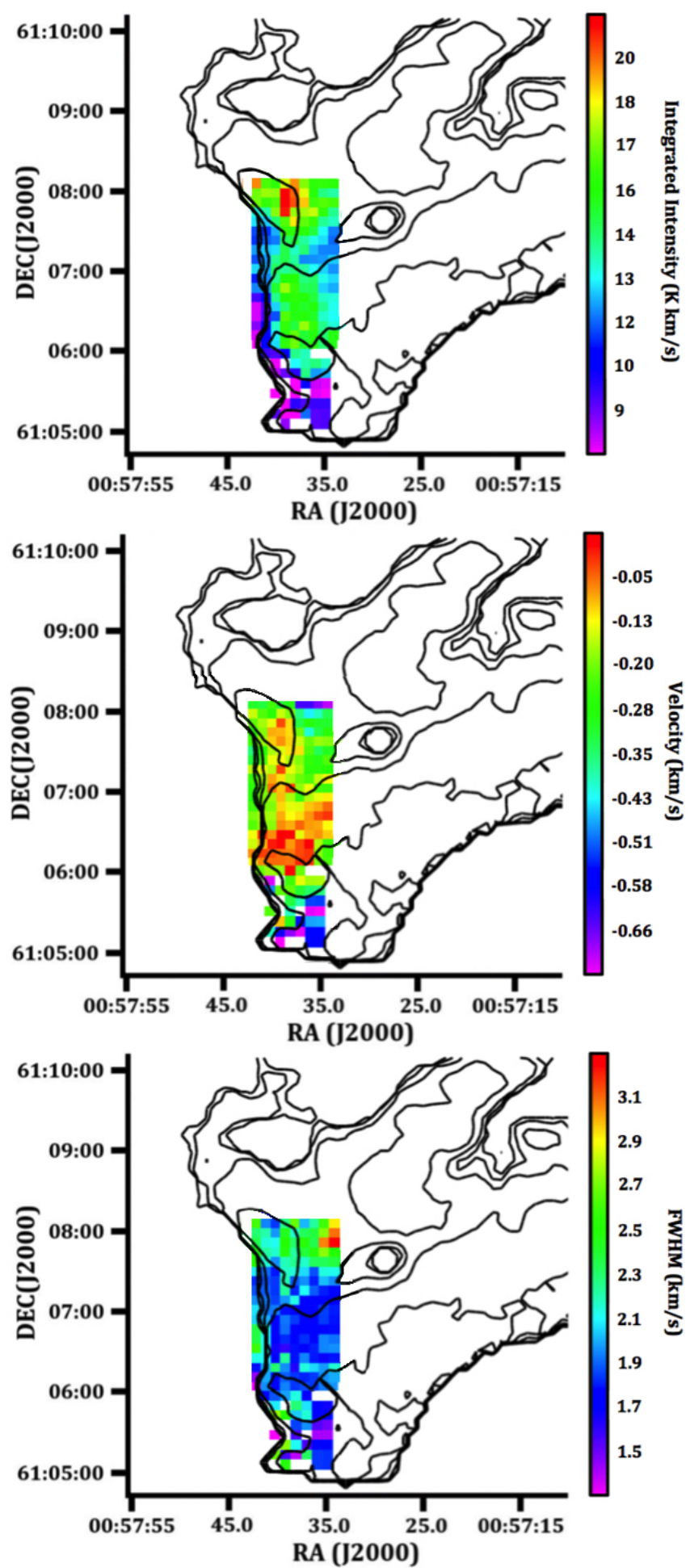

Fig. 9. SOFIA results on the [C II] $157 \mu \mathrm{m}$ line emission in IC 59. The panels show the maps of the central velocity of the line (middle panel), the FWHM of the [C II] lines after a Gaussian line profile fit (bottom panel), and the integrated line intensity (top panel). The black contours correspond to the contours of the dust temperature shown in Fig. 6, and are plotted for visual guidance purposes only.

from a visual inspection of the correlation plots. This zone corresponds to a region farther away from the star, already in the dense cloud where the PAH emission is weaker. The PAH band ratios in this zone alone do not vary much, that is greater variations in the PAH band ratios are observed across all zones in 

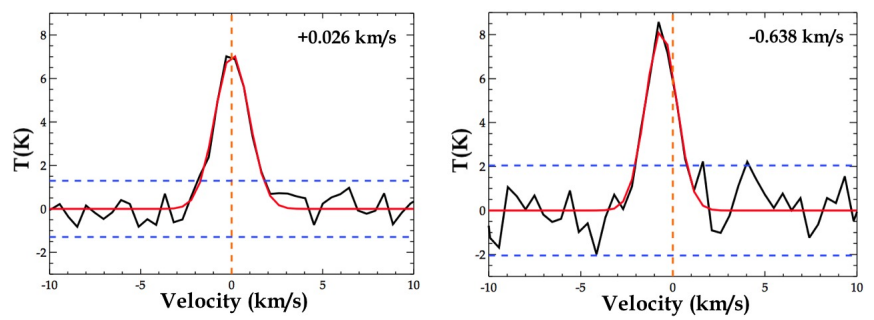

Fig. 10. Example spectra of the [C II] $157 \mu \mathrm{m}$ line for two different positions in IC 59. The data is shown in black, while the Gaussian line profile fit is shown in red. The blue dashed lines indicate $\pm 2 \sigma$ of the continuum level. Right panel: spectrum at the position $(\mathrm{RA}, \mathrm{Dec})=$ $(00: 57: 37,+61: 08: 07)$ at the northern part of the map shown in Fig. 9, left panel: corresponds to a position in the southern part of the map, at $(\mathrm{RA}, \mathrm{Dec})=(00: 57: 39,+61: 06: 28)$.

Table 5. Summary of physical conditions at the tip of IC 63 and IC 59.

\begin{tabular}{ccc}
\hline \hline Property & IC 63 & IC 59 \\
\hline$G_{0}$ & 150 & 25 \\
$n\left(\mathrm{~cm}^{-3}\right)$ & $1.3 \times 10^{4}$ & $2 \times 10^{3}$ \\
$T_{\text {gas }}(\mathrm{K})$ & 207 & 150 \\
$T_{\text {dust }}(\mathrm{K})$ & 30 & 27 \\
$\epsilon_{\text {gas }}$ & 0.016 & 0.018 \\
$G_{0} / n$ & 0.012 & 0.013 \\
$\gamma$ & 1079 & 995 \\
\hline
\end{tabular}

NGC 7023, as such region is extended enough to trace variations in the physical conditions.

The similarity of IC 63 (and IC 59) PAH band ratios with those from zone 5 of NGC 7023 is consistent with its similarity to the far region mentioned in Sect. 3.1 (see also Fig. 5). The PAH band ratios found for IC 63 (and IC 59) compared to other sources then, resemble (or are even more extreme than) the ratios found in the more shielded parts of other reflection nebulae such as NGC 7023 and NGC 2023.

\subsection{Variability of PAH emission}

The observed variations in relative intensities of the main PAH bands are mainly controlled by the PAH charge balance. This in turn is set by the competition between the ionization and recombination rates, and is thus defined by the so-called ionization parameter $\gamma \equiv G_{0} \sqrt{T_{\text {gas }}} / n_{\mathrm{e}}$, where $n_{\mathrm{e}}$ is the electron density. We have estimated $\gamma$ at the tip of IC 63 and IC 59 assuming that, as expected in PDRs, the electrons come from the ionization of neutral carbon atoms, and thus we adopt a carbon abundance of $1.6 \times 10^{-4}$ (Sofia et al. 2004). The derived values are listed in Table 5. Based on the physical conditions we derived for IC 63 and IC 59, we find that the ionization parameter, as well as $G_{0} / n$ are similar for these two regions. The similarity between their PAH emission is thus not surprising.

The ionization parameter is an indicator of the ionization degree of the emitting PAHs (Bregman \& Temi 2005; Galliano et al. 2008; Stock et al. 2016). Galliano et al. (2008) studied several Galactic regions for which the physical conditions were known, and for which the IR spectra were observed with either ISO or Spitzer telescopes. Based on this data set, they obtained an empirical relation between the ionization parameter and the 6.2/11.3 PAH band ratio (see Fig. 11). The data points for IC 63

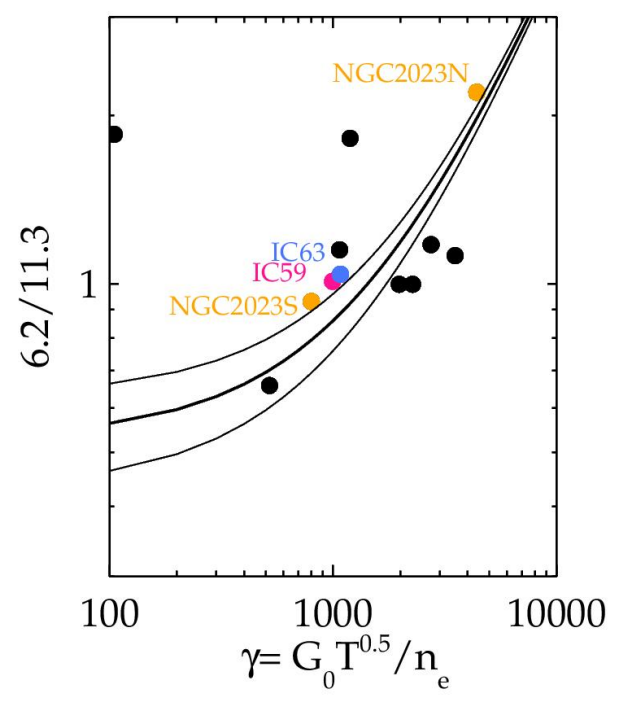

Fig. 11. Ionization parameter $\gamma$ versus the 6.2/11.3 PAH band ratio. The data points for IC 63 and IC 59 are shown in blue and red, respectively. The black points are taken from Stock et al. (2016), while the lines correspond to the fit and its $1 \sigma$ deviations derived from the systematic study of PAH emission in Galactic regions done by Galliano et al. (2008). We have highlighted the data points for NGC 2023 N (north) and NGC 2023 S (south), since these are PDRs in the reflection nebula NGC 2023, which is a relatively benign environment comparable to IC 63 and IC 59.

and IC 59 agree with the relation found by Galliano et al. (2008) within $3 \sigma$, and lie close to the data points of $\mathrm{H}$ II regions and other reflection nebulae such as NGC 2023 (Stock et al. 2016). In fact we again see the average $6.2 / 11.3$ is close to that of NGC 2023 south (S), which is the denser than the northern PDR, NGC 2023 N.

\subsection{Heating efficiency}

As seen from the relation depicted in Fig. 11, the higher the ionization parameter, the greater the 6.2/11.3 PAH ratio, which is expected to trace the ionization degree of the emitting PAHs. The charge state of PAHs is important in determining the efficiency of the photoelectric effect (Bakes \& Tielens 1994). The heating efficiency of the gas $\epsilon_{\text {gas }}$ can be obtained by comparing the emissions of the cooling lines with the dust continuum emission. Assuming that all of the cooling of the gas in the PDR is done through the [C II] 157 and [O I] $63 \mu \mathrm{m}$ fine structure lines, we can approximate $\epsilon_{\text {gas }}$ as the ratio $([\mathrm{CII}]+[\mathrm{OI}]) / I_{\mathrm{FIR}}$. In this expression $I_{\mathrm{FIR}}$ corresponds to the total far-IR flux and we calculate it from the SEDs. The results are shown in Fig. 12 as well as in Table 5. The derived heating efficiencies $\epsilon_{\text {gas }}$ for IC 63 and IC 59, appear to be consistent with an inverse relation between $\epsilon_{\text {gas }}$ and $\gamma$, as the more ionized the PAHs, the less effective is the photoelectric heating of the gas.

The efficiencies found at the tip of IC 63 and IC 59 are comparable to those found in the relatively low-UV excited PDRs of the Horsehead Nebula $\left(\epsilon_{\text {gas }} \sim 2 \%, G_{0} \sim 100\right.$; Goicoechea et al. 2009). Our observational estimates of $\epsilon_{\text {gas }}$ also agree with the theoretical predictions from Bakes \& Tielens (1994), where the theoretical efficiencies are expected to be $\sim 2 \%$ for both IC 63 and IC 59 considering the $\gamma$ values listed in Table 5 (see Eq. (40) in Bakes \& Tielens 1994). It is important to keep in mind that the $\gamma$ value determined here is a rather rough estimate, as it involves an assumption on the electron density, and an estimate of the 


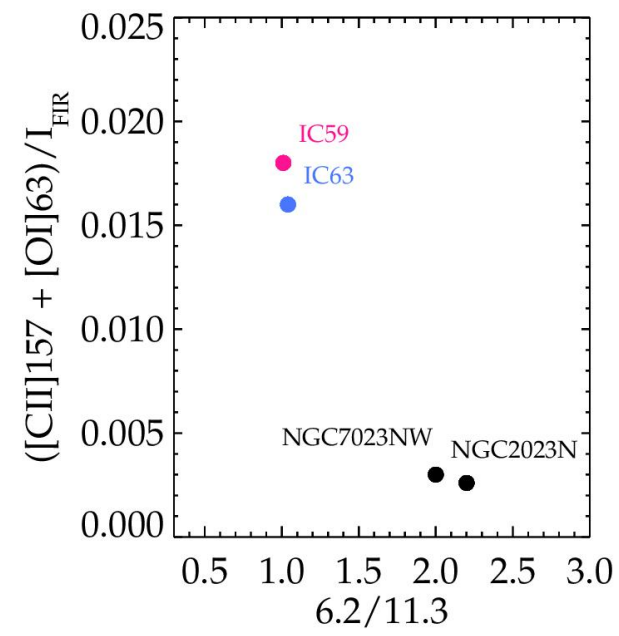

Fig. 12. Comparison between the 6.2/11.3 PAH band ratio and the heating efficiency approximated by the ([C II $] 157+[\mathrm{OI}] 63) / I_{\mathrm{FIR}}$ ratio. The data points for IC 63 and IC 59 are shown in the same colors as in the previous figures. The data points for the northwestern PDR of NGC 7023 (Boersma et al. 2014; Bernard-Salas et al. 2015) and the north PDR of NGC 2023 (Stock et al. 2015; Peeters et al. 2017) are shown in black for comparison.

gas temperature of the region. Observations in other PDRs have found heating efficiencies derived from observables tend to be lower than the theoretical predictions by no more than a factor of two (Okada et al. 2013; Pabst et al. 2017). However, the inverse relation between heating efficiency and $\gamma$ still applies.

\subsection{Other correlations}

Stock \& Peeters (2017) found an empirical relation between the intensity ratio of the two components of the $7.7 \mu \mathrm{m}$ PAH band, and the UV radiation field given in Habing field units. As their sample consisted mostly on high- $G_{0}$ PDRs, it is of great interest to plot IC 63 and IC 59 data points to sample the low- $G_{0}$ end of the relation. We therefore decomposed the $7-9 \mu \mathrm{m}$ region following Stock \& Peeters (2017). The $7.7 \mu \mathrm{m}$ PAH band is fitted with two Gaussian components at 7.55 and $7.87 \mu \mathrm{m}$, with fixed peak wavelength and FWHM. The results are shown in Fig. 13. Since the $7.8 \mu \mathrm{m}$ band has been associated with PAHs with irregular structures, while the $7.6 \mu \mathrm{m}$ component has been ascribed to more compact (and thus stable) species (Peeters et al. 2017), the inverse relation between the 7.8/7.6 and $G_{0}$ might be tentatively reflecting the destruction of irregularshaped (less stable) PAHs in high-UV irradiated environments. In addition to structural changes, it is hypothesized that the $7.8 \mu \mathrm{m}$ component originates in larger species, and thus this ratio may also reflect changes in the size distribution of its carriers (Peeters et al. 2017). Given the derived $G_{0}$ values for IC 63 and IC 59, it is clear that they agree with the relations found by Stock \& Peeters (2017) in the low- $G_{0}$ limit. Interestingly enough, our derived 7.8/7.6 ratios fall close to the value found in the Horsehead PDR, and differ greatly from the higher- $G_{0}$ PDRs of NGC 2023 N and NGC 7023 NW, in consistency with Figs. 7, 11 and 12 .

Likewise, Pilleri et al. (2012) reported an inverse correlation between $G_{0}$ and the fraction of carbon atoms locked in the so-called evaporating very small grains (eVSGs) relative to the carbon in the PAHs, $f_{\mathrm{eVSG}}$. This was interpreted as PAHs being released into space by the photo-evaporation of

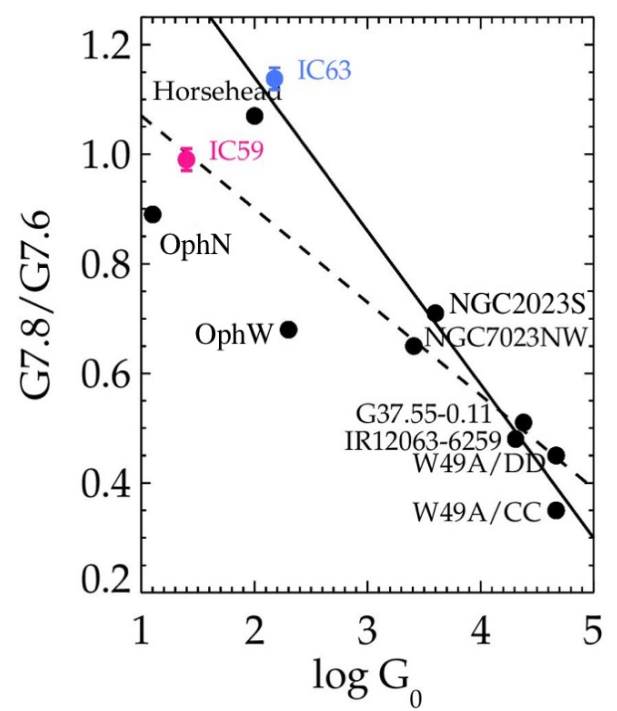

Fig. 13. Intensity ratio of the two components of the $7.7 \mu \mathrm{m}$ PAH band versus the intensity of the UV radiation field $G_{0}$. Again the data points for IC 63 and IC 59 are shown in the same colors as in the previous figures. The black points are taken from Stock \& Peeters (2017). The dashed line corresponds to the fit to all black data points, while the solid line corresponds to the fit excluding the data points in the Ophiuchus diffuse cloud (Stock \& Peeters 2017).

VSGs in high-UV radiation environments, in agreement with previous observational studies (Rapacioli et al. 2005; Berné et al. 2007). The fraction of eVSGs was determined by using the fitting tool PAHTAT (PAH Toulouse Astronomical Templates; Pilleri et al. 2012) which yields the relative contributions of different dust emission components for a given IR spectrum, which are positively ionized PAHs, neutral PAHs and the eVSG component. We applied PAHTAT to the average spectra of IC 63 and IC 59. For IC 63, we found $f_{\mathrm{eVSG}}=0.72$, which is consistent with the estimate published by Pilleri et al. (2012), who found $f_{\text {eVSG }}=0.63$ (data point labeled IC 63(P) in Fig. 14). This, together with our new estimate of $G_{0}$, situates IC 63 in the upper left corner of the $G_{0}$ versus $f_{\mathrm{eVSG}}$ diagram, also in good agreement with their published relation (see Fig. 14).

For IC 59, we found a $f_{\text {evSG }}=0.60$ which does not appear to be consistent with the observed relation (Fig. 14), unless we are underestimating $G_{0}$ by a factor greater than $\sim 100$, which seems unlikely, at least at the tip of the nebula. We note that Pilleri et al. (2012) explicitly stated the relation is applicable for $10^{2} \lesssim G_{0} \lesssim$ $10^{4}$ and densities in the range $10^{2}-10^{5} \mathrm{~cm}^{-3}$ as observations and modeling are difficult outside these ranges.

\subsection{Morphology of IC 59}

Figure 2 shows the general structure of IC 63 and IC 59 as seen in different wavelengths. Bright $\mathrm{H} \alpha$ rims delineate the IRAC $8.0 \mu \mathrm{m}$ and PACS $160 \mu \mathrm{m}$ emission for both nebulae. In addition to the bright $\mathrm{H} \alpha$ rims in IC 63, faint extended $\mathrm{H} \alpha$ emission is also seen at the tip of the nebula (where the IRS spectra have been taken). This is also what we see at the tip of IC 59. Only farther away from the star, clear bright clumps are seen in the IRAC $8.0 \mu \mathrm{m}$ image (around position $(\mathrm{RA}, \mathrm{Dec})=$ $(00: 57: 20,+61: 10: 00))$. In this sense, for both nebulae we find that the regions where the PAH emission is the brightest are not where the IRS SL-LL datasets have been observed. 


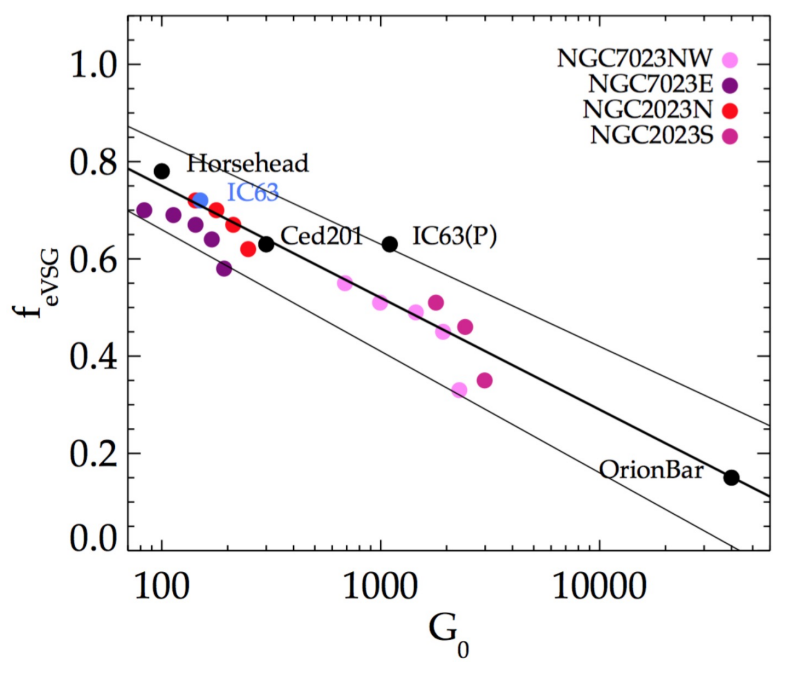

Fig. 14. Fraction of carbon atoms locked in eVSGs relative to the carbon in the PAHs, $f_{\mathrm{evsG}}$, versus UV field intensity, $G_{0}$. Data points and the correlation have been taken from Pilleri et al. (2012). We have highlighted the data points for the PDRs in the reflection nebulae NGC 7023 and NGC 2023 for comparison purposes with IC 63. The data point IC 63(P) corresponds to the estimate obtained by Pilleri et al. (2012), which assumed a $G_{0}=1100$ corresponding to the 650 Draine fields published in Jansen et al. (1994).

In particular, for IC 59 the tip of the nebula (as seen from optical images) is not the region most intensely hit by the stellar radiation (see Figs. 2 and 6). This instead happens to the western concave side of the nebula, which lies facing the radial direction to the star. This explains why the PAH and [C II] emission at the tip is weak compared to the northern region. The $G_{0}$ value found from the SED indicates the tip is indeed farther away from the star, and thus it is mostly a projection effect. We encourage then follow up studies to focus on the northwestern region of IC 59 rather than the tip.

A comparison of the velocity of [C II] $157 \mu \mathrm{m}$ with that of the ionized gas and molecular gas can tell us about the dynamics of the region as influenced by the incident stellar radiation, as [C II] is an important tracer of PDRs. Previous studies on the kinematics of the entire nebula of IC 59 report that the molecular gas (as traced by ${ }^{12} \mathrm{CO}(J=1-0)$ ) shows velocities between +1.03 and $+2.65 \mathrm{~km} \mathrm{~s}^{-1}$, peaking at about $2 \mathrm{~km} \mathrm{~s}^{-1}$ (Karr et al. 2005). Such peak velocity is observed in an unresolved clump of molecular material observed farther north from the tip of IC 59. The position of this clump corresponds to the brightest position in the IR bands shown in Fig. 2 (IRAC $8 \mu \mathrm{m}$ and PACS $160 \mu \mathrm{m}$ images). We suspect that this is the material behind the ionization front produced by $\gamma \mathrm{Cas}$ that is hitting the western concave part of IC 59 (and that this material is closer to the star than the tip where the IRS-SL data was taken).

Our [C II] $157 \mu \mathrm{m}$ velocity map shows velocities between -0.74 and $+0.33 \mathrm{~km} \mathrm{~s}^{-1}$. Previous measurements on neutral gas, as traced by the $21 \mathrm{~cm}$ emission, show velocities of about $1 \mathrm{~km} \mathrm{~s}^{-1}$ (Blouin et al. 1997, Karr et al. 2005). Fich et al. (1990) determined a velocity for the ionized gas of $-2 \mathrm{~km} \mathrm{~s}^{-1}$ for IC 63 . Assuming this to be valid also for IC 59, the data would be suggesting an overall velocity gradient between the different components, consistent with an interface being photo-evaporated due to the radiation from $\gamma$ Cas. The material lying almost perpendicular to the radial direction to the star (where the [C II] velocity map shows a redshifted component that crosses the map in the southeast to northwest direction) appears to be "dragged" by the incident radiation of the star (Fig. 9). Adopting an estimate of the mass of the cloud of IC 59 equal to $m_{\mathrm{c}}=0.7 M_{\odot}$ (Blouin et al. 1997) we can obtain the evaporation timescale of the cloud $t_{\mathrm{pe}}$, considering the mass loss rate $\mathrm{d} m_{\mathrm{c}} / \mathrm{d} t \sim A_{\mathrm{c}} \times \rho_{\mathrm{PDR}} \times c_{\mathrm{PDR}}$, where $A_{\mathrm{c}}$ corresponds to the area facing the ionization front produced by the radiation of $\gamma$ Cas; $\rho_{\text {PDR }}$ corresponds to the mass density of the heated gas; and $c_{\mathrm{PDR}}$ is the isothermal sound speed in the PDR gas. Considering a kinetic temperature of $650 \mathrm{~K}$, we obtain a speed of $2 \mathrm{~km} \mathrm{~s}^{-1}$. Assuming a facing area $\sim \pi r_{\mathrm{c}}^{2}$, with the radius $r_{\mathrm{c}} \sim 0.3 \mathrm{pc}$, and a density $\sim 10^{-22} \mathrm{gr} \mathrm{cm}^{-3}$, we obtain a rough estimate for the mass loss rate of $\sim 10^{-6} M_{\odot} \mathrm{yr}^{-1}$. This would imply a time scale of $\sim 1$ Myr for the evaporation of the available reservoir of gas in the nebula.

\section{Conclusion}

We re-visit the pair of nebulae IC 63 and IC 59, both being cometary-shaped clouds in the vicinity of a single star, $\gamma$ Cas. Using Herschel and Spitzer photometry and spectroscopy, and high resolution velocity maps from SOFIA, we studied the PAH emission at the tip of both nebulae, and we make an assessment of the general physical conditions under which they emit.

We found that the PAH emission, as quantitatively measured in terms of PAH band ratios, is remarkably homogeneous throughout the tip of IC 63. The ratios found are also quite similar to the PAH band ratios derived from the average spectrum of the tip of IC 59. This we claim to be due to a similar $G_{0} / n$ ratio and ionization parameter in the two objects, as derived from the photometric data and cooling line ratios.

Comparison to other well-studied nebulae, the $\mathrm{PAH}$ band ratios found for IC 63 and IC 59 ratios are similar to those found in the more shielded regions of other nebulae, such as NGC 7023 and NGC 2023. Considering the physical conditions derived in this work, we get that the PAH emission observed in IC 63 and IC 59 consistently situates these nebulae as low- $G_{0}$ PDRs, contrary to what has been predicted in previous studies.

Using SOFIA data we obtained [C II] $157 \mu$ m velocity maps for IC 59. Given the cometary shape of IC 59 and the velocity gradient between the molecular gas, $\mathrm{H} \mathrm{I}$ and [C II] components, we claim that the cloud is being photo-evaporated due to the influence of the star. The difference in the morphology of IC 59 observed in the optical compared to the IR wavelengths, suggests that the tip (as seen in optical images) of IC 59 is quite likely to be farther away from the star than the rest of the cloud, and that the cometary shape of IC 59 is mostly the result of projection effects.

Acknowledgements. Based (in part) on observations made with the NASADLR Stratospheric Observatory for Infrared Astronomy (SOFIA). SOFIA is jointly operated by the Universities Space Research Association, Inc. (USRA), under NASA contract NAS2-97001, and the Deutsches SOFIA Institut (DSI) under DLR contract 50 OK 0901 to the University of Stuttgart. E.P. acknowledges support from an NSERC Discovery Grant. Studies of interstellar PAHs at Leiden Observatory are supported through advanced ERC grant 246976 from the European Research Council, through a grant by the Dutch Science Agency, NWO, as part of the Dutch Astrochemistry Network, and through the Spinoza premie from the Dutch Science Agency, NWO.

\section{References}

Allamandola, L. J., Tielens, A. G. G. M., \& Barker, J. R. 1985, ApJ, 290, L25 Allamandola, L. J., Hudgins, D. M., \& Sandford, S. A. 1999, ApJ, 511, L115 Andersson, B.-G., Piirola, V., De Buizer, J., et al. 2013, ApJ, 775, 84 Andrews, H., Boersma, C., Werner, M. W., et al. 2015, ApJ, 807, 99 Bakes, E. L. O., \& Tielens, A. G. G. M. 1994, ApJ, 427, 822 Barentsen, G., Farnhill, H. J., Drew, J. E., et al. 2014, MNRAS, 444, 3230 
Bauschlicher, Jr. C. W., Peeters, E., \& Allamandola, L. J. 2008, A\&A, 678, 316 Bernard-Salas, J., Habart, E., Köhler, M., et al. 2015, A\&A, 574, A97

Berné, O., Joblin, C., Deville, Y., et al. 2007, A\&A, 469, 575

Blouin, D., McCutcheon, W. H., Dewdney, P. E., et al. 1997, MNRAS, 287, 455

Boersma, C., Rubin, R. H., \& Allamandola, L. J. 2012, ApJ, 753, 168

Boersma, C., Bauschlicher, Jr. C. W., Ricca, A., et al., 2014, ApJS, 211, 8

Bregman, J., \& Temi, P. 2005, ApJ, 621, 831

Bron, E., Le Petit, F., \& Le Bourlot, J. 2016, A\&A, 588, A27

Cantalupo, C. M., Borrill, J. D., Jaffe, A. H., Kisner, T. S., \& Stompor, R. 2010, ApJS, 187, 212

Code, A. D., \& Meade, M. R. 1979, ApJS, 39, 195

Compiègne, M., Flagey, N., Noriega-Crespo, A., et al. 2010, ApJ, 724, L44

Drew, J. E., Greimel, R., Irwin, M. J., et al. 2005, MNRAS, 362, 753

Dupac, X., Bernard, J., Boudet, N., et al. 2003, A\&A, 404, L11

D’Hendecourt, L. B., \& Léger, A. 1987, A\&A, 180, 9

Fadda, D., Jacobson, J. D., \& Appleton, P. N. 2016, A\&A, 594, A90

Fazio, G. G., Hora, J. L., Allen, L. E., et al. 2004, ApJS, 154, 39

Fich, M., Dahl, G. P., \& Treffers, R. 1990, AJ, 99, 622

Fleming, B., France, K., Lupu, R. E., \& McCandliss, S. R. 2010, ApJ, 725, 159

France, K., Andersson, B.-G., McCandliss, S. R., \& Feldman, P. D. 2005, ApJ, 628,750

Fuente, A., Martín-Pintado, J., Rodríguez-Fernández, N. J. et al. 1999, ApJ, 482, L45

Galliano, F., Madden, S. C., Tielens, A. G. G. M., Peeters, E., \& Jones, A. P. 2008, ApJ, 679, 310

Goicoechea, J. R., Compiégne, M., \& Habart, E. 2009, ApJ, 699, L165

Gordon, K. D., Engelbracht, C. W., Rieke, G. H., et al. 2008, ApJ, 682, 336

Griffin, M. J., Abergel, A., Abreu, A., et al. 2010, A\&A, 518, L3

Habart, E., Boulanger, F., Verstraete, L. et al. 2003, A\&A, 397, 623

Habart, E., Abergel, A., Boulanger, F., et al. 2011, A\&A, 527, A122

Heyminck, S., Graf, U. U., Güsten, R., et al. 2012, A\&A, 542, L1

Hollenbach, D. J., \& Tielens, A. G. G. M. 1997, ARA\&A, 35, 179

Hollenbach, D. J., \& Tielens, A. G. G. M. 1999, Rev. Mod. Phys., 71, 173

Hollenbach, D. J., Takahashi, T., \& Tielens, A. G. G. M. 1991, ApJ, 377, 192

Hony, S., Van Kerckhoven, C., Peeterset, E. et. al. 2001, A\&A, 370, 1030

Houck, J. R., Roellig, T. L., van Cleve, J., et al. 2004, ApJS, 154, 18

Jansen, D. J., van Dishoeck, E. F., \& Black, J. H. 1994, A\&A, 282, 605

Jansen, D. J., van Dishoeck, E. F., Black, J. H., Spaans, M., \& Sosin C. 1995, A\&A, 302, 223

Jansen, D. J., van Dishoeck, E. F., Keene, J., Boreiko, R. T., \& Betz, A. L. 1996, A\&A, 309, 899

Karr, J. L., Noriega-Crespo, A., \& Martin, P. G. 2005, AJ, 129, 954

Kaufman, M. J., Wolfire, M. G., Hollenbach, D. J., \& Luhman, M. L. 1999, ApJ, 527,795

Kaufman, M. J., Wolfire, M. G., \& Hollenbach, D. J. 2006, ApJ, 644, 283

Köhler, M., Habart, E., Arab, H., et al. 2014, A\&A, 569, A109
Langhoff, S. R. 1996, J. Phys. Chem. 100, 2819

Léger, A., \& Puget, J. L. 1984, A\&A, 137, L5

Lepp, S., \& Dalgarno, A. 1988, ApJ, 335, 769

Markwardt, C. B. 2009, in Astronomical Data Analysis Software and Systems XVIII, eds. D. A. Bohlender, D. Durand, \& P. Dowler (San Francisco, CA: ASP), 411, 251

Meixner, M., Haas, M. R., Tielens, A. G. G. M., Erickson, E. F., \& Werner, M. 1992, ApJ, 390, 499

Ochsendorf, B. B., \& Tielens, A. G. G. M. 2015, A\&A, 576, A2

Okada, Y., Pilleri, P., Berné, O., et al. 2013, A\&A, 553, A2

Pabst, C. H. M., Goicoechea, J. R., Teyssier, D., et al. 2017, A\&A, 606, A29

Peeters, E., Hony, S., Van Kerkhoven, C., et al. 2002, A\&A, 390, 1089

Peeters, E., Tielens, A. G. G. M., Allamandola, L. J., \& Wolfire, M. G. 2012, ApJ, 747, 44

Peeters, E., Bauschlicher, C. W., Allamandola, L. J., et al. 2017, ApJ, 836 198

Pety, J. 2005, Semaine de l'Astrophysique Française, ed. F. Casoli, et al., EDP Sciences, p. 721

Pilbratt, G. L., Riedinger, J. R., Passvogel, T., et al. 2010, A\&A, 518, L1

Pilleri, P., Montillaud, J., Berné, O., \& Joblin, C. 2012, A\&A, 542, A69

Poglitsch, A., Waelkens, C., Geis, N., et al. 2010, A\&A, 518, L2

Pound, M., \& Wolfire, M. G. 2008, ADASS XVII, 394, 654

Rapacioli, M., Joblin, C., \& Boissel, P. 2005, A\&A, 429, 193

Rieke, G. H., Young, E. T., Engelbracht, C. W., et al. 2004, ApJS, 154, 25

Rosenthal, D., Bertoldi, F., \& Drapatz, S. 2000, A\&A, 356, 705

Sellgren, K., Uchida, K. I., \& Werner, M. W. 2007, ApJ, 659, 1338

Shannon, M. J., Stock, D. J., \& Peeters, E. 2015, ApJ, 811, 153

Smith, J. D. T., Draine, B. T., Dale, D. A., et al. 2007, ApJ, 656, 770

Soam, A., Maheswar, G., Lee, C. W., Neha, S., \& Andersson, B.-G. 2017, MNRAS, 465, 559

Sofia, U. J., Lauroesch, J. T., Meyer, D. M., \& Cartledge, S. I. B. 2004, ApJ, 605, 272

Stock, D. J., \& Peeters, E. 2017, ApJ, 837, 129

Stock, D. J., Wolfire, M. G., Peeters, E., et al. 2015, A\&A, 579, A67

Stock, D. J., Choi, W. D.-Y., Moya, L. G. V., et al. 2016, ApJ, 819, 65

Swinyard, B. M., Ade, P., Baluteau, J.-P., et al. 2010, A\&A, 518, L4

Temi, P., Marcum, P. M., Young, E., et al. 2014, ApJS, 212, 24

Thi, W.-F., van Dishoeck, E. F., Bell, T., Viti, S., \& Black, J. 2009, MNRAS 400,622

Tielens, A. G. G. M. 2005, The Physics and Chemistry of the Interstellar Medium (Cambridge: Cambridge University Press)

Tielens, A. G. G. M., \& Hollenbach, D. 1985, ApJ, 291, 747

Troy, B. E., Jr. Johnson, C. Y., Young, J. M., \& Holmes, J. C. 1975, ApJ, 195, 643

Vakili, F., Granes, P., Bonneau, D., Noguchi, M., \& Hirata, R. 1984, PASJ, 36, 231

Werner, M. W., Uchida, K. I., Sellgren, K., et al. 2004, ApJS, 154, 309

Young, E. T., Becklin, E. E., Marcum, P. M., et al. 2012, ApJ, 749, L17 